\title{
The Effectiveness of Mindfulness-Based Interventions on Maternal Perinatal Mental Health Outcomes: a Systematic Review
}

\author{
Zhenrong Shi ${ }^{1} \cdot$ Angus MacBeth $^{1}$ (D) \\ Published online: 19 January 2017 \\ (C) The Author(s) 2017. This article is published with open access at Springerlink.com
}

\begin{abstract}
Presenting with common mental health difficulties, particularly depression and anxiety, there is also preliminary evidence that mindfulness-based interventions (MBIs) including mindfulness-based cognitive therapy (MBCT), mindfulnessbased stress reduction (MBSR) and integrated mindfulness yoga practices may also be effective in reducing common mental health difficulties during pregnancy. We systematically reviewed and synthesized the current literature on the effectiveness of MBIs in reducing severity of perinatal anxiety and depression. Databases including PubMed, Cochrane Library, IndMED and PsychoInfo were searched for relevant studies. Manual searches were conducted in relevant articles and Google Scholar. Seventeen cohorts representing 18 studies were included. Prepost effect sizes were reported for both treatment and control groups. Seven randomized controlled trials (RCTs), two nonrandomized controlled trials and nine treatment evaluations were included. Maternal participation in an MBI was associated with reductions in perinatal anxiety of moderate to large magnitude. Results for the effect of MBIs on depression were less consistent, with pre-post treatment reductions of moderate magnitude, but no significant differences in depression scores when MBI was compared with a control group. There was some evidence that MBIs were associated with increased mindfulness. Risk of bias in studies was variable. Our review offers preliminary evidence for the effectiveness of MBIs in reducing perinatal anxiety, with more equivocal findings with regard to perinatal
\end{abstract}

Angus MacBeth

angus.macbeth@ed.ac.uk

1 Clinical and Health Psychology, School of Health in Social Science, Old Medical Quad, University of Edinburgh, Scotland, UK depressive symptoms. Further methodologically rigorous evaluation using RCTs and longer follow-up periods are recommended.

Keywords Mindfulness-based interventions .

Mindfulness-based cognitive therapy · Mindful-based .

Stress reduction $\cdot$ Mindfulness yoga $\cdot$ Perinatal $\cdot$ Anxiety Depression · Pregnancy

\section{Introduction}

Pregnancy and the postnatal period is a time of rapid and significant change in a women's life, encompassing biological, social and psychological changes. Although often a time of positive emotion, estimates of the prevalence of anxiety and depression suggest at least $10 \%$ of pregnant women experience perinatal anxiety (Andersson et al. 2006), 20\% of pregnant women suffer prenatal depression and between 12 and $16 \%$ of pregnant women are likely to suffer postnatal depression (Leung and Kaplan 2009). There is also substantial comorbidity between perinatal anxiety and depression (Di Florio et al. 2013; Grigoriadis et al. 2011). For instance, elevated anxiety during pregnancy is also considered as a risk factor for postpartum depression (e.g. Sutter-Dallay et al. 2004). Consequently, reducing perinatal psychological distress (anxiety, depression and stress occurring during pregnancy or in the first-year post-pregnancy) is a crucial public health goal.

There is increasing evidence that perinatal anxiety, depression and stress have both short- and long-term negative effects on mothers and newborns, with additional complex interactions between these variables (Staneva et al. 2015a). Both maternal anxiety, depression and stress increase risks for adverse neonatal outcomes including preterm birth (e.g. Dole et al. 2003; Glynn et al. 2008) and low birth weight (Dunkel Schetter and Lobel 
2012; Hoffman and Hatch 2000) and small fetal head size (Field et al. 2010). These adverse outcomes are themselves linked to increased risk of infant mortality, neurodevelopmental impairment and disabilities (Wilson-Costello 2005) and adverse physical and mental health outcomes in later life (Sydsjö 2011). In addition, perinatal depression and anxiety may impact psychological development via changes to mother-infant interactions (e.g. Nicol-Harper et al. 2007), language development of children at 12 months (Quevedo et al. 2012) and behavioural, emotional and cognitive problems in middle childhood (Glover and O'Connor 2006; Huizink et al. 2003; O'Connor et al. 2002). From a treatment perspective, perinatal anxiety and depression may also be under-detected and untreated (Goodman and TyerViola 2010). Furthermore, although antidepressant medication is effective in treating anxiety and depression, there is evidence of possible side effects of medication on neonatal outcomes including low birth weight, preterm birth, low Apgar scores, respiratory distress, neonatal convulsions and hypoglycemia (e.g. Grigoriadis et al. 2014; Hendrick et al. 2003; Huang et al. 2014; Kallen 2004). These risks indicate that development of effective non-pharmacological interventions in pregnancy would be beneficial.

A large number of studies suggest mindfulness-based interventions (MBIs) such as Mindfulness-based stress reduction (MBSR; Kabat-Zinn 2003) and Mindfulness-based cognitive therapy (MBCT; Segal et al. 2002) are effective psychological interventions to reduce depression and anxiety in clinical and non-clinical populations (Kuyken et al. 2015). MBIs have demonstrated effectiveness in preventing the recurrence of depression (Piet and Hougaard 2011; Segal et al. 2002), and MBSR has demonstrated effectiveness in reducing symptoms of both generalized anxiety (e.g. Hoge et al. 2013) and social anxiety (Koszycki et al. 2007). A recent meta-analytic review (Hoffman et al. 2010) reported a moderate effect size of MBIs on anxiety and mood reduction for all participants and a strong effect size for reducing anxiety $(g=0.97)$ and $\operatorname{mood}(g=0.95)$ symptoms for those participants with pre-existing anxiety and mood disorders.

In addition, there is also an emergent evidence base for mindfulness-informed yoga interventions in pregnancy. With regard to general health in pregnancy, yoga integrated with a meditation intervention has been demonstrated to improve maternal physical health in pregnancy and improve labor and birth outcomes (Curtis et al. 2012; Narendran et al. 2005). There is also evidence that yoga practice in pregnancy reduces perinatal anxiety and depression (Newham et al. 2014). It is of note that non-pharmacologic interventions in pregnancy such as yoga and MBIs share overlapping common characteristics such as meditation and regulated breathing. Cramer et al. (2013) suggested that yoga and meditation may have effectiveness in the treatment of mental health difficulties. With specific reference to pregnancy, Gong et al. (2015) reviewed evidence that integrated yoga-including physical exercises, breathing (pranayama), meditation or deep relaxation - was effective in reducing prenatal depression. However, the results did not demonstrate the effectiveness of physical-exercise-based yoga. In addition, Beddoe et al. (2010) demonstrated that women in the third trimester reported significant anxiety and stress reductions after receiving mindfulness-informed yoga. Therefore, the evidence base for MBIs in perinatal mental health pregnancy could be enriched by considering yoga interventions that explicitly integrate mindfulness practice with yoga techniques (Muzik et al. 2012).

In summary, there is preliminary evidence that MBIs may be effective to anxiety and depression reduction for pregnant women (e.g. Vieten and Astin 2008, Woolhouse et al. 2014) and similar preliminary evidence regarding the effectiveness of yoga on reducing distress in pregnancy (Beddoe et al. 2010). There have been meta-analyses of mindfulness interventions in pregnancy (Hall et al. 2016; Taylor et al. 2016), focused on outcomes in common mental health symptoms, with both reviews highlighting issues with the quality of the data. However, these reviews varied in their approach to study designs, assessment of risk of bias and definitions of MBIs (including MBSR, MBCT and mindfulness-informed yoga). The literature on mindfulness in pregnancy also continues to accumulate at a rapid pace. We sought to systematically review the evidence for the effectiveness of MBIs (MBCT, MBSR and mindfulness-informed yoga) on common mental health difficulties (specifically anxiety, depression and stress) in pregnancy, with a focus on a narrative synthesis of the theoretical and methodological challenges in the current literature. Specifically, we hypothesized that MBIs would be effective in reducing levels of depression and anxiety both from pre-post treatment and compared to controls. We also hypothesized that there would be a broad range of methodological variance in the literature.

\section{Method}

The review was conducted according to PRISMA guidelines (Moher et al. 2015. Four electronic bibliographic databases (PubMed, Cochrane Library, Ended and PsychInfo) were searched up to 28 September 2016. Database limits were set from 1980 to September 2016. Search terms were combined from conjunctions of the following terms: ('mindfulness' OR 'mindfulness techniques' OR 'mindfulness approaches' OR mindfulness-based cognitive therapy' OR 'mindfulness-based interventions/ or treatments' OR 'MBCT' OR 'mindfulnessbased stress reduction' OR 'mindful yoga' OR 'mindful meditation') AND ('perinatal depression' OR 'peripartum depression' OR 'maternal depression' OR 'antenatal depression' OR 'prenatal depression' OR 'pre-partum depression' OR 'postpartum depression' OR 'postnatal depression') OR ('perinatal anxiety' OR 'peripartum anxiety' OR 'maternal anxiety', OR 
'antenatal anxiety'). Manual searches were conducted for cross-references in relevant articles and review papers extracted from the database searches and in Google Scholar by using the combination of the above terms. An expert librarian was consulted with regard to the search terms.

Our inclusion criteria were for female participants meeting the following criteria: participants were either primigravida or multigravida; measurement of depression and/or anxiety symptoms was implemented using either validated selfreport or interview measures; or participants met diagnostic criteria for a depressive or anxiety disorder based on criteria from the Diagnostic and Statistical Manual, Fourth Edition (DSM-IV) (American Psychiatric Association 2000) or the International Classification of Disease 10 (ICD-10) criteria (World Health Organization, 1993). Participants were assessed either during pregnancy or during first year after delivery, aged between 16 and 45 years old and could speak and read English. In addition, studies were selected if they compared MBI with a control group (either treatment without therapist, treatment as usual or care as usual or waiting-list control conditions) or without a control group. Study designs were either randomized controlled trials (RCTs), nonrandomized controlled trials, or non-controlled trials with quantitative data. Finally, studies were included if their treatment component used either manualized protocols or accredited facilitators or was delivered by health professional with specific training in facilitation of MBIs.

Studies were excluded if participants had current psychosis or other complex mental disorders, where depressive and/or anxiety symptoms were comorbid symptoms of a specific physical disorder and where women were a priori identified as medically defined high-risk pregnancies (e.g. multiple pregnancies). In addition, we excluded qualitative studies, case studies, book chapters and literature reviews. No restrictions were made in terms of the participants' attendance rate of the mindfulness-based interventions, ethnic origin, marital status, weeks of gestation and previous experience of MBIs.

Interventions were eligible for inclusion if they included an MBI such as MBSR or MBCT. We included yoga interventions only where there was clear evidence from the intervention description that the intervention included several components consistent with integrated mindfulness practice (e.g. techniques to encourage a non-judgemental focus on sensation experienced in the current moment, meditation, breathing, body scan, deep relaxation), rather than simply a description of yoga practices per se. We therefore included studies with an explicit statement that the yoga intervention included integrated mindfulness practice. Interventions without detailed description of its components were excluded. No restrictions were made regarding the length, frequency or duration of the MBI. Included studies had to examine at least one of the primary outcomes: anxiety and depression. Secondary outcomes of interest were stress and mindfulness. Inclusion of studies was initially made by the first author. Where there was uncertainty regarding inclusion, queries were resolved by consensus discussion with the second author.

An adaption of the SIGN 50 Methodology Checklist (Scottish Intercollegiate Guideline Network checklist 2015) was used to extract study characteristics including specific details about the study design, population, interventions, followup, outcome measures and results. For studies which providing the mean scores and standard deviations of baseline as well as post-interventions, effect size (ES) Cohen's $d$ and their 95\% confidence intervals were calculated. For controlled studies, ES was calculated for the differences between pre- and postinterventions in both the treatment and control groups. For non-controlled studies, ES was also calculated in treatment group to compare the changes from baseline to post-interventions. Effect sizes (ESs; Cohen 1988) were divided into five levels: trivial $(d \leq 0.2)$, small $(d>0.2)$, moderate $(d>0.5)$, large $(d>0.8)$, and very large $(d>1.3)$. The revised Cochrane risk of bias tool was used to evaluate risk of bias of included studies (Higgins et al. 2011). The studies were rated according to five domains: selection bias, performance bias, detection bias, attrition bias and reporting bias. The first author assessed the risk of bias for all studies. Inter-rater reliability was calculated by the second rating of a randomly selected $40 \%$ of studies by an independent investigator, blind to review aims. The inter-rater reliability was 0.80 (Cohen's kappa), indicating high agreement between the two reviewers on risk of bias assessments.

\section{Results}

Procedures for screening of studies are displayed in Fig. 1. The final data set consisted of 17 studies reporting results from $k=18$ cohorts (Fig. 1). One study (Woolhouse et al. 2014) reported results from two distinct samples (hereafter labelled samples 1 and 2). A summary of study characteristics of the 18 included cohorts is presented in Table 1. Of the included studies, seven studies were randomized controlled trials (Dimidjian et al. 2016; Guardino et al. 2014; Narimani and Musavi 2015; Perez-Blasco et al. 2013; Vieten and Astin 2008; Woolhouse et al. 2014, sample 1; Zhang and Emory, 2015); two studies were nonrandomized controlled trials (Dunn et al. 2012; Miklowitz et al. 2015) and nine studies were non-controlled trials (Battle et al. 2015; Beddoe et al. 2010; Byrne et al. 2014; Dimidjian et al. 2015; Duncan and Bardacke 2010; Felder et al. 2016; Goodman et al. 2014; Muzik et al. 2012; Woolhouse et al. 2014, sample 2). Of the included five RCTs, the control group types were waiting-list control $(n=2$; Perez-Blasco et al. 2013; Vieten and Astin 2008); care-as-usual $(n=4$; Dimidjian et al. 2016; Dunn et al. 2012; Woolhouse et al. 2014, sample 1; Zhang and Emory, 2015); no intervention ( $n=1$; Narimani and Musavi $2015)$ and reading control ( $n=1$; Guardino et al. 2014). 
Fig. 1 PRISMA flow chart for identification and selection of studies

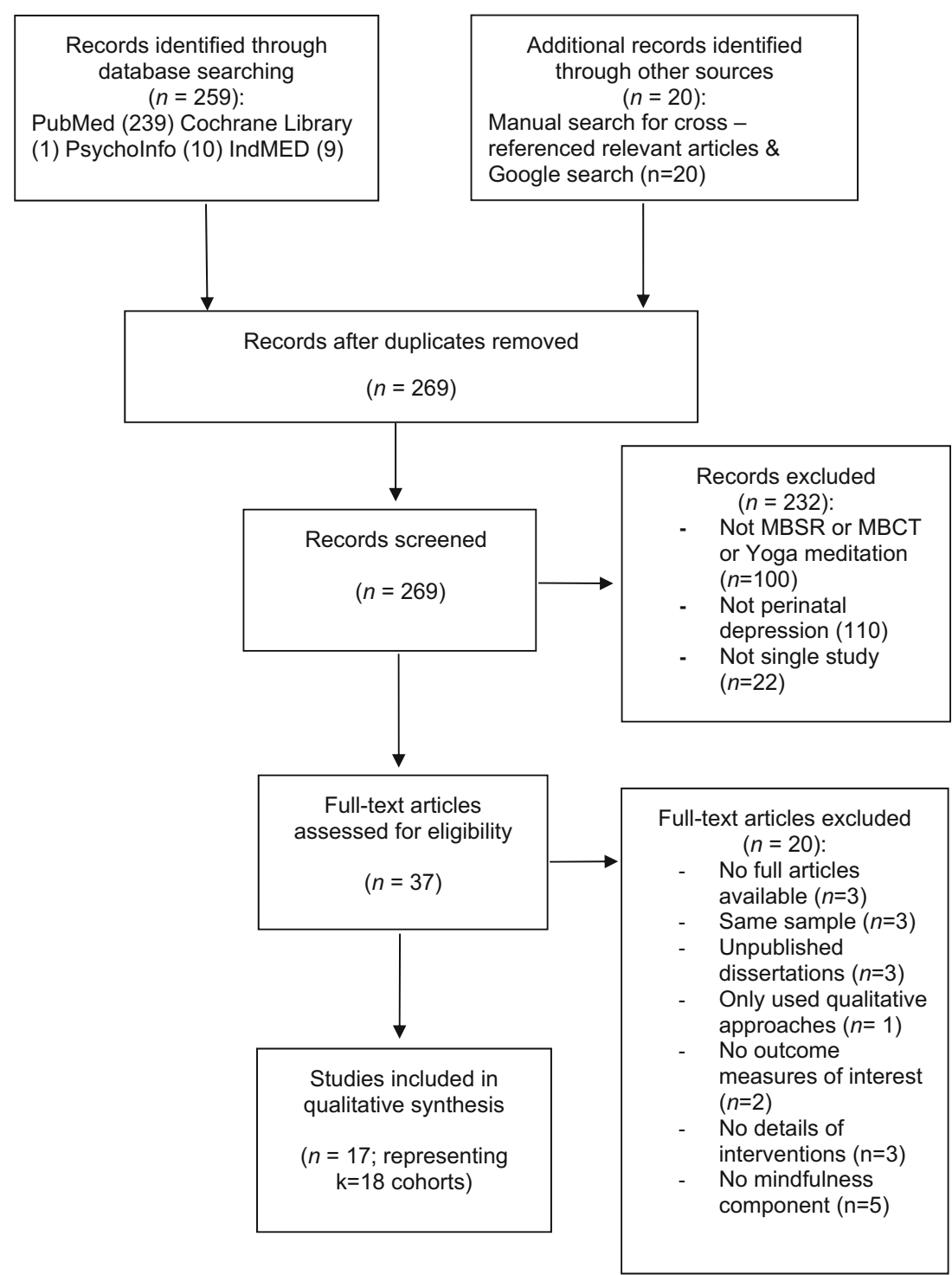

In terms of study settings and participant characteristics, there were $n=640$ participants enrolled in the included studies. After excluding participants who dropped out of the intervention programs or failed to finish post-intervention assessments, findings from $n=603$ participants were reported. Twelve studies were conducted in the USA (Battle et al. 2015; Beddoe et al. 2010; Dimidjian et al. 2015, 2016; Duncan and Bardacke 2010; Felder et al. 2016; Goodman et al. 2014; Guardino et al. 2014; Miklowitz et al. 2015; Muzik et al. 2012; Vieten and Astin 2008; Zhang and Emory 2015); four studies in Australia (Byrne et al. 2014; Dunn et al. 2012; Woolhouse et al. 2014, both samples); one in Iran (Narimani and Musavi 2015) and one in Spain (PerezBlasco et al. 2013). Of the included studies, sixteen studies involved adult pregnant women, one study involving pregnant adolescents who were less than 20 years old and one recruited women in the first-year post-pregnancy. All included studies reported the mean age; most studies involved adults with mean maternal age ranged from 30 to 35 years old $(n=15)$. Fifteen studies reported the mean gestation at the start of the intervention, while three studies did not report the details of gestation. Across studies, the mean gestation ranged from the first trimester to middle 3rd trimester. Nine studies involved mental health samples of participants who were experiencing or identified at risk of stress, anxiety and/ or depression. Eight studies involved participants who were non-depressed and anxious, healthy women recruited from the general population, although three of these studies involved screening for mental health difficulties. One study targeted women from low-income, ethnic minority areas (Zhang and Emory 2015). With the exception of two studies (Narimani and Musavi 2015; Zhang and Emory 2015), all studies reported the majority of participating women to be married, cohabiting or living together. Studies were conducted in a variety of settings from university clinics, maternity hospitals and general clinics. 


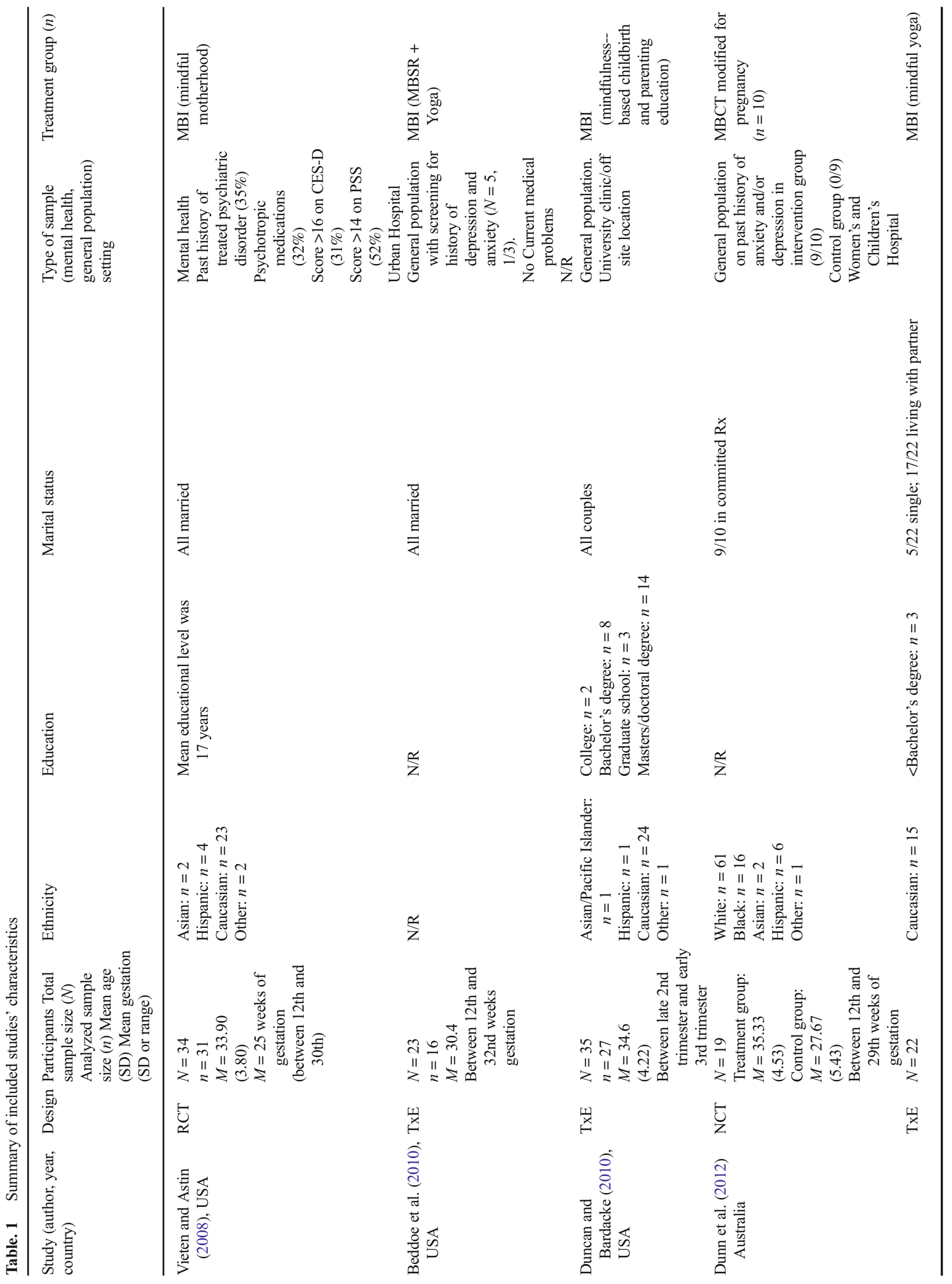




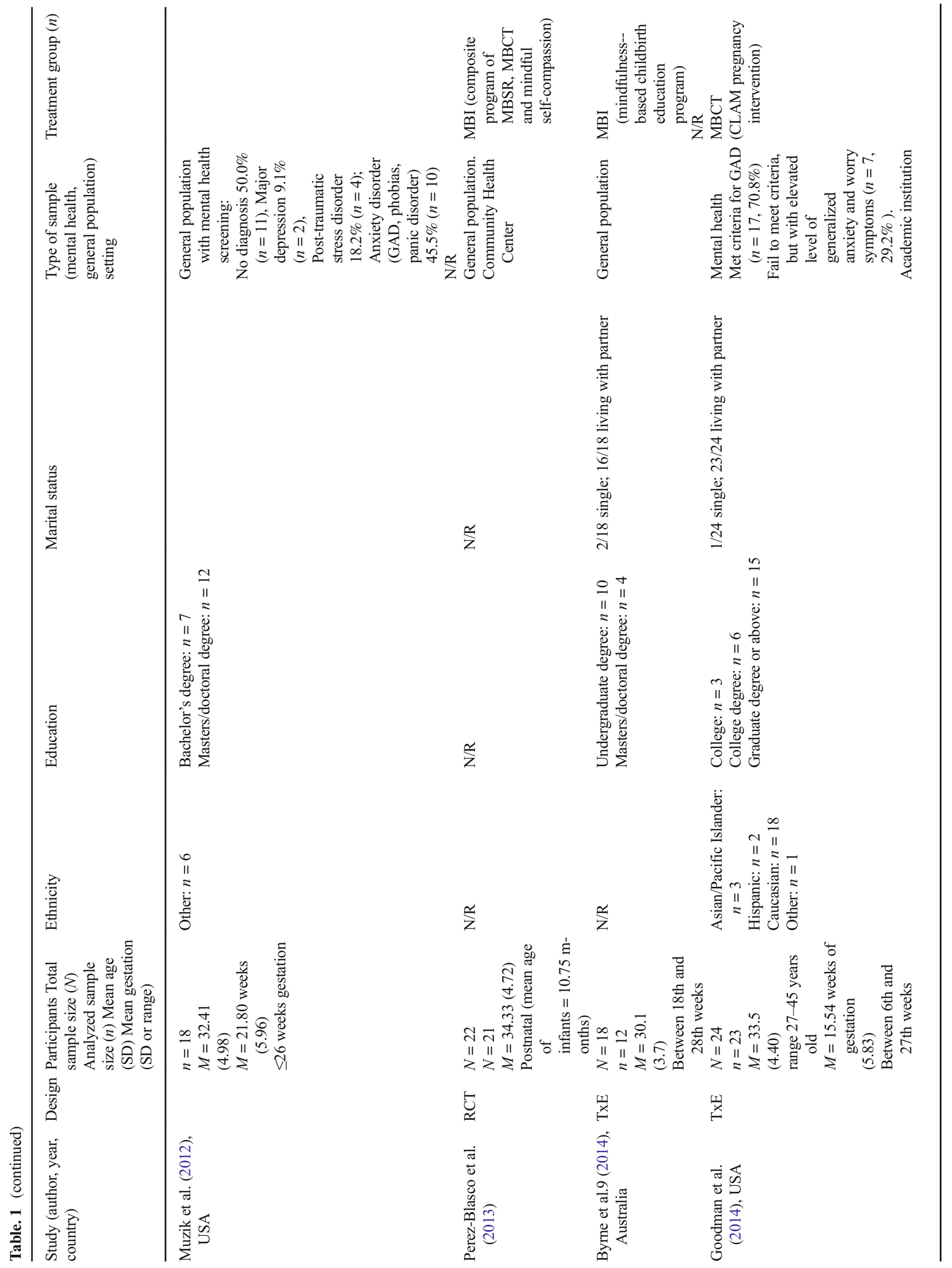




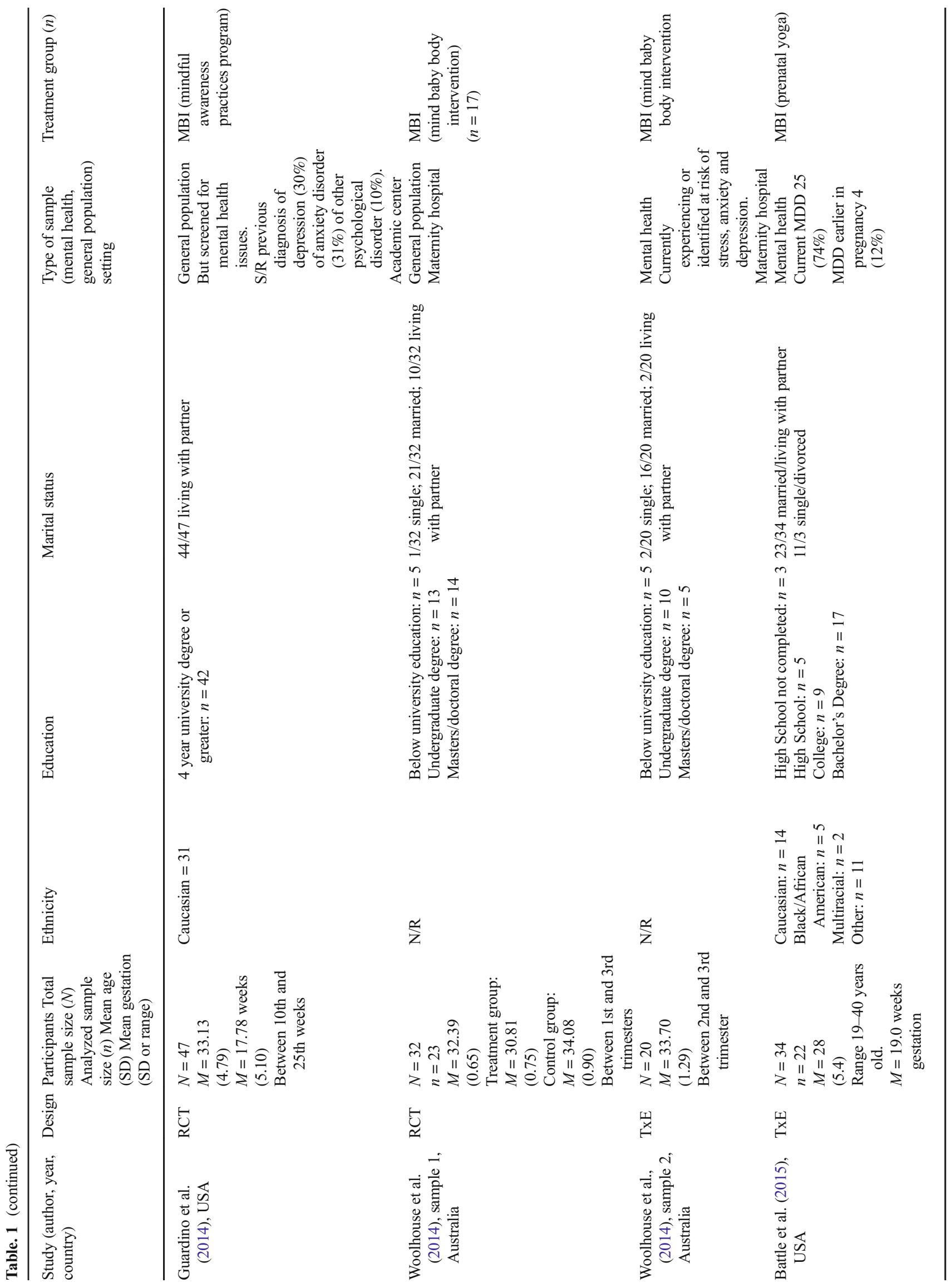




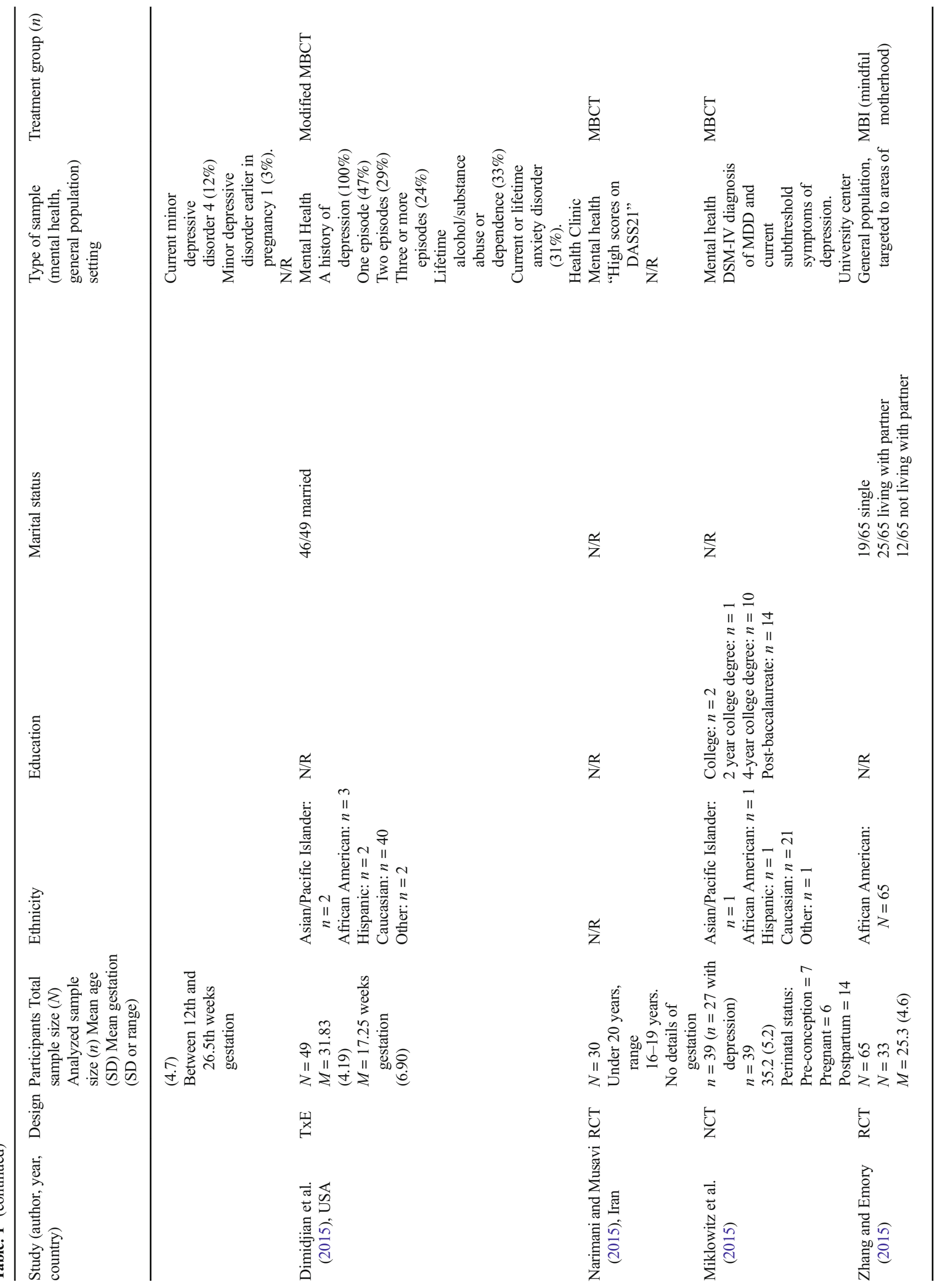




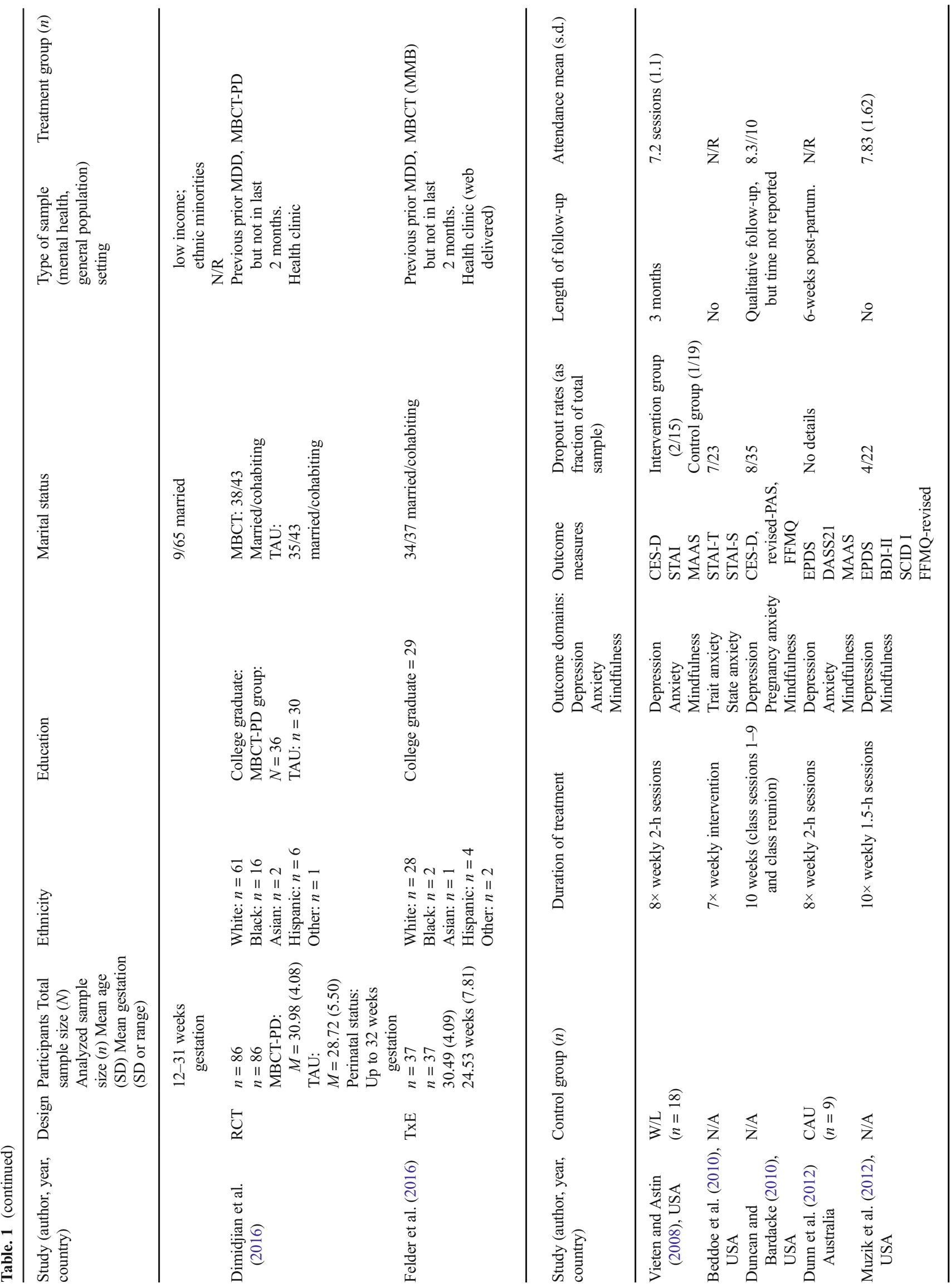




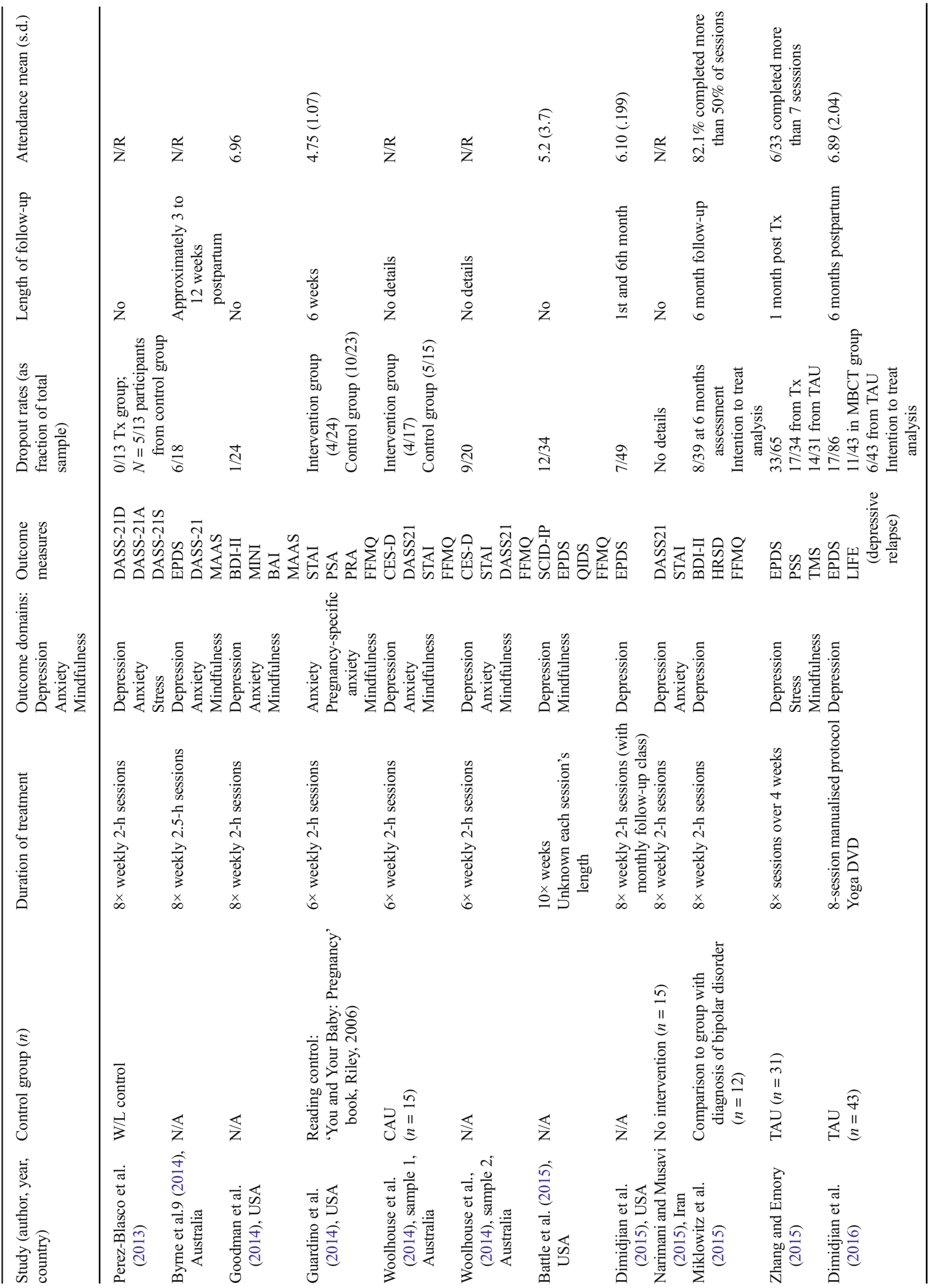




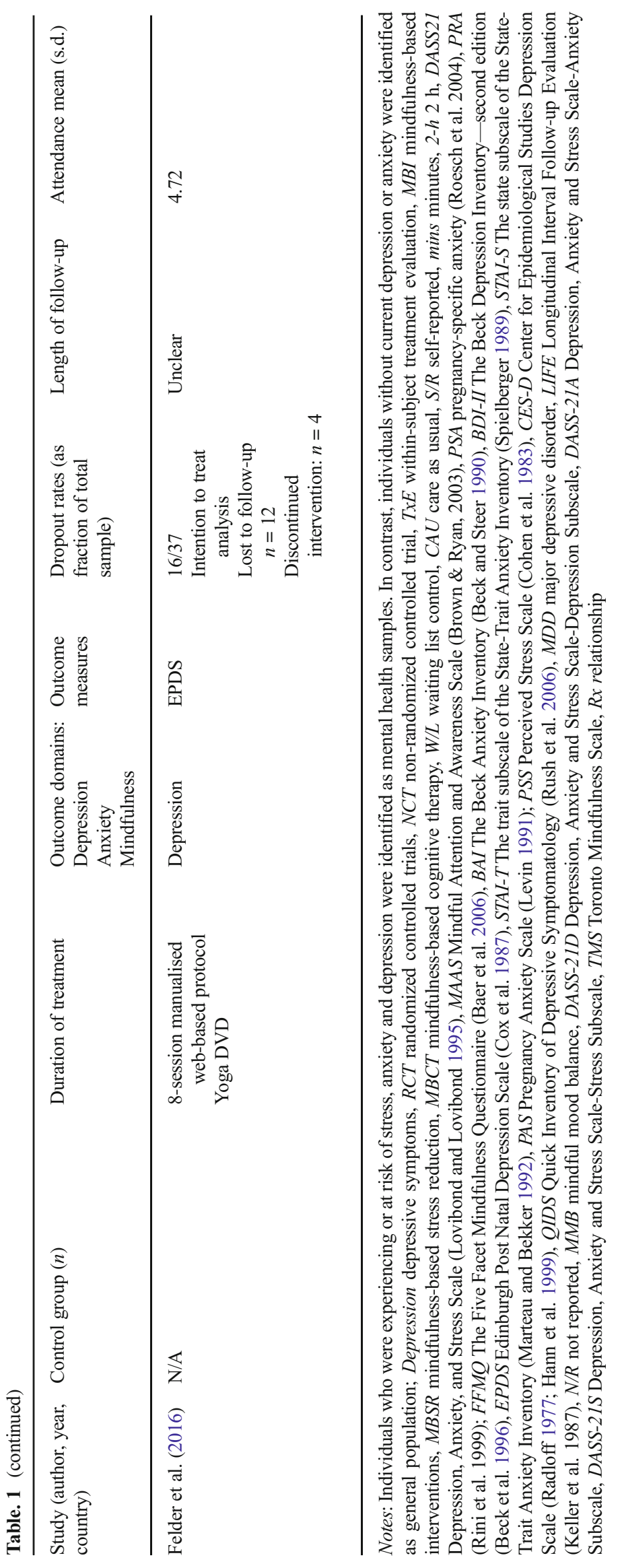


With regard to treatment identified MBIs included variants on MBCT, MBSR and mindfulness yoga. Seven studies used MBCT (Dimidjian et al. 2015, 2016; Dunn et al. 2012; Felder et al. 2016; Goodman et al. 2014; Miklowitz et al. 2015; Narimani and Musavi 2015). Nine studies used MBSR or variants (Guardino et al. 2014; Vieten and Astin 2008; Woolhouse et al. 2014, both samples; Beddoe et al. 2010; Byrne et al. 2014; Duncan and Bardacke 2010; Perez-Blasco et al. 2013; Zhang and Emory 2015). One study involved mindfulness yoga (Muzik et al. 2012), and the other one used prenatal yoga (Battle et al. 2015). Mean duration of treatment was 8 weeks (range $=6$ to 10 weeks). The mean session length was $2 \mathrm{~h}$ (range $=1.5$ to $2.5 \mathrm{~h}$ ). All sessions were led by trained instructors, clinical psychologists or certificated therapists. Engagement with treatment in most studies was high, particularly for MBCT- and MBSR-based approaches. One study (Zhang and Emory 2015) reported low levels of engagement throughout the treatment program.

The outcome measures used for assessment of depression and anxiety varied between studies. All included studies used self-report measures to assess depression and anxiety symptoms. The 18 included studies conducted baseline assessment and immediate post-treatment assessment. In addition, eight studies conducted post-treatment follow-up assessments. The timing of assessment ranged from 3 weeks to 6 months postpartum.

The effectiveness of MBIs upon depressive symptoms was examined in 16 studies (Table 2). With regard to controlled studies, of the six RCTs, three showed significant posttreatment reductions in depressive symptoms for MBCT compared to controls (Dimidjian et al. 2016; Narimani and Musavi 2015; Zhang and Emory 2015). Two RCTs showed trends toward post-treatment improvement for MBIs, based on selfreport measures of depression (Vieten and Astin 2008; Woolhouse et al. 2014, sample 1). One study showed no difference between groups (Perez-Blasco et al. 2013). In the nonrandomized trials, one study (Dunn et al. 2012) reported clinically significant different scores with inconclusive results for reduction in depression, while one study reported reductions in depressive symptoms for MBCT (Miklowitz et al. 2015). For within-group changes, the four RCTs, two reported large ESs $(d=0.70$; Dimidjian et al. 2016; $d=0.83$; Perez-Blasco et al. 2013) and three reported small to moderate ESs ( $d=0.53$; Vieten and Astin 2008; $d=0.30$ and $d=0.54$ for CES-D and DASS-32, respectively; Woolhouse et al. 2014, sample 1; Zhang and Emory 2015).

In eight non-controlled studies, significant improvements were reported for depressive symptoms after completing MBIs (Battle et al. 2015; Dimidjian et al. 2015; Duncan and Bardacke 2010; Goodman et al. 2014; Muzik et al. 2012; Woolhouse et al. 2014, sample 2). Two non-controlled studies did not find significant reductions in depressive symptoms (Byrne et al. 2014; Felder et al. 2016). Most studies reported moderate to large ESs (Byrne et al. 2014; Dimidjian et al. 2015; Goodman et al. 2014; Muzik et al. 2012; Woolhouse et al. 2014, sample 2), while one study showed a small ES (Duncan and Bardacke 2010) and one showed a negligible effect (Byrne et al. 2014, $d=0.08$ ).

With regard to anxiety, 12 studies examined the effectiveness of MBIs on anxiety symptoms (Table 3 ). Included RCTs $(n=7)$ suggested that participants engaging with MBIs showed significant reductions in anxiety compared with controls (all $p<.05$, Guardino et al. 2014; Narimani and Musavi 2015; Perez-Blasco et al. 2013; Vieten and Astin 2008; Woolhouse et al. 2014, sample 1). One non-randomized controlled study reported that one out of four participants was free of anxiety symptoms after treatment compared to none in the control group (Dunn et al. 2012). Of the five controlled studies, three RCTs reported data convertible to ESs. Most of the effects were of moderate to large size (Guardino et al. 2014; Perez-Blasco et al. 2013; Vieten and Astin 2008; Woolhouse et al. 2014, sample 1). In the one study that assessed pregnancy-related anxiety, there was a small effect size (Guardino et al. 2014). Four out of five non-controlled studies suggested significant improvements of anxiety after treatment (Beddoe et al. 2010; Duncan and Bardacke 2010; Goodman et al. 2014; Woolhouse et al. 2014, sample 2). However, two studies reported reductions in anxiety that did not reach statistical significance (Byrne et al. 2014; Woolhouse et al. 2014, sample 2), although in one study, a large ES was observed (Woolhouse et al. 2014, sample 2). These studies both used the DASS-21 to measure anxiety. Three studies showed large ESs, while one showed a small to moderate $\operatorname{ES}(d=0.31)$ (Byrne et al. 2014).

Of the included studies, six RCTs, one non-randomized controlled study and four non-controlled studies assessed pre- to post-treatment changes in stress (Table 2). Withinsubject ESs suggested large pre-post ESs for reduction in stress (three studies) (Guardiano et al. 2014; Perez Blasco et al. 2013; Vieten and Astin 2008), and two studies suggested reductions of moderate magnitude (Woolhouse et al. 2014, sample 1, DASS-21; Zhang and Emory 2015). However, one study reported a small effect using the Perceived Support Scale (PSS; Woolhouse et al. 2014, sample 1). One study reported data that could not be converted to give ESs (Narimani and Musavi 2015). Results were more equivocal when MBIs were measured against a control. Here, only one study suggested a significant effect favoring MBI (PerezBlasco et al. 2013). In addition, the one non-randomized controlled study (Dunn et al. 2012) reported $75 \%$ of the treatment group reported a clinically reliable reduction in stress, while none of the control participants showed reductions in stress. In terms of outcomes from non-controlled studies, the results are similarly equivocal. One study (Beddoe et al. 2010) reported a significant decrease in perceived stress over time $(p=.05)$, which they proposed was related to the third trimester group. 


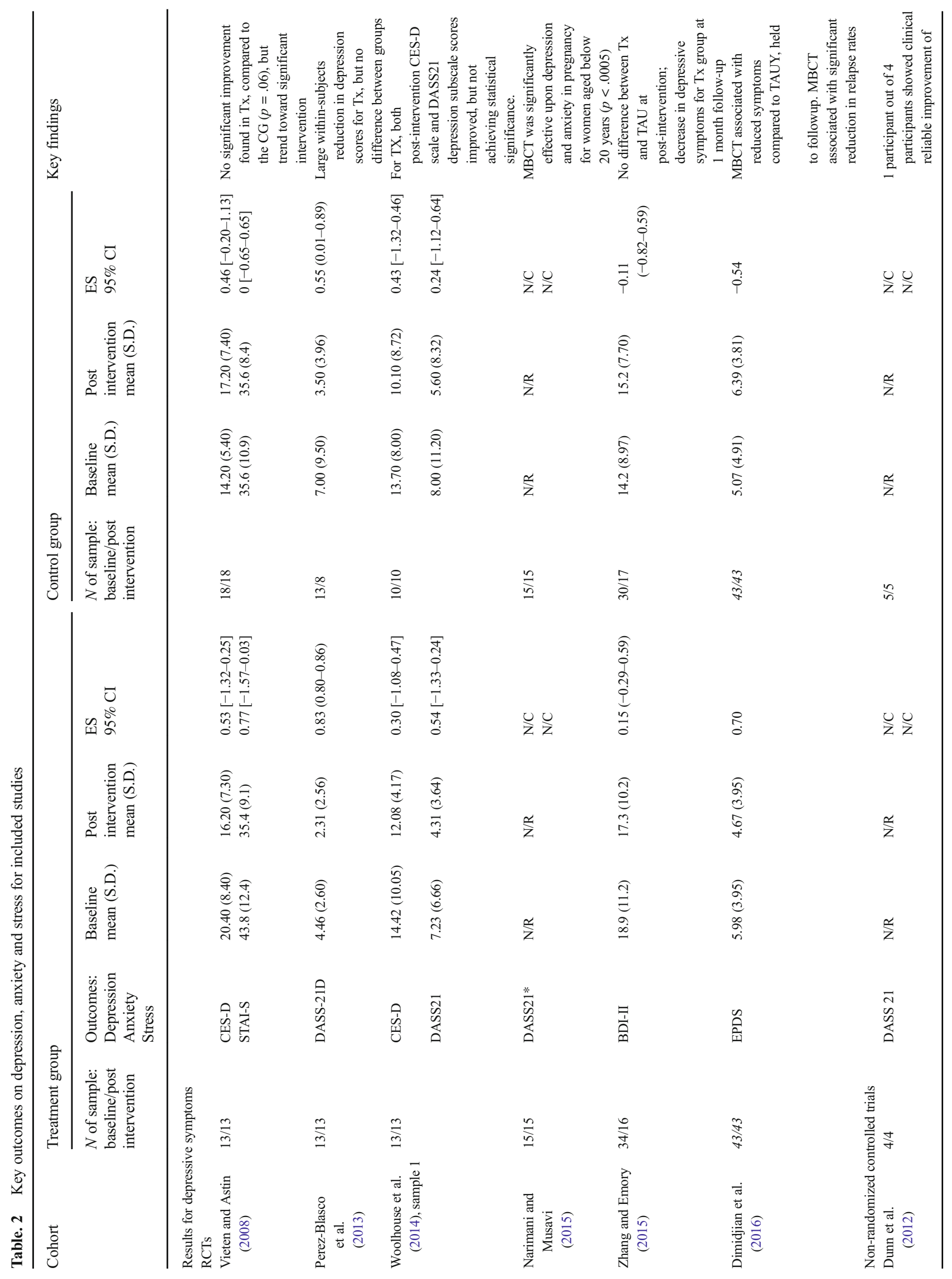




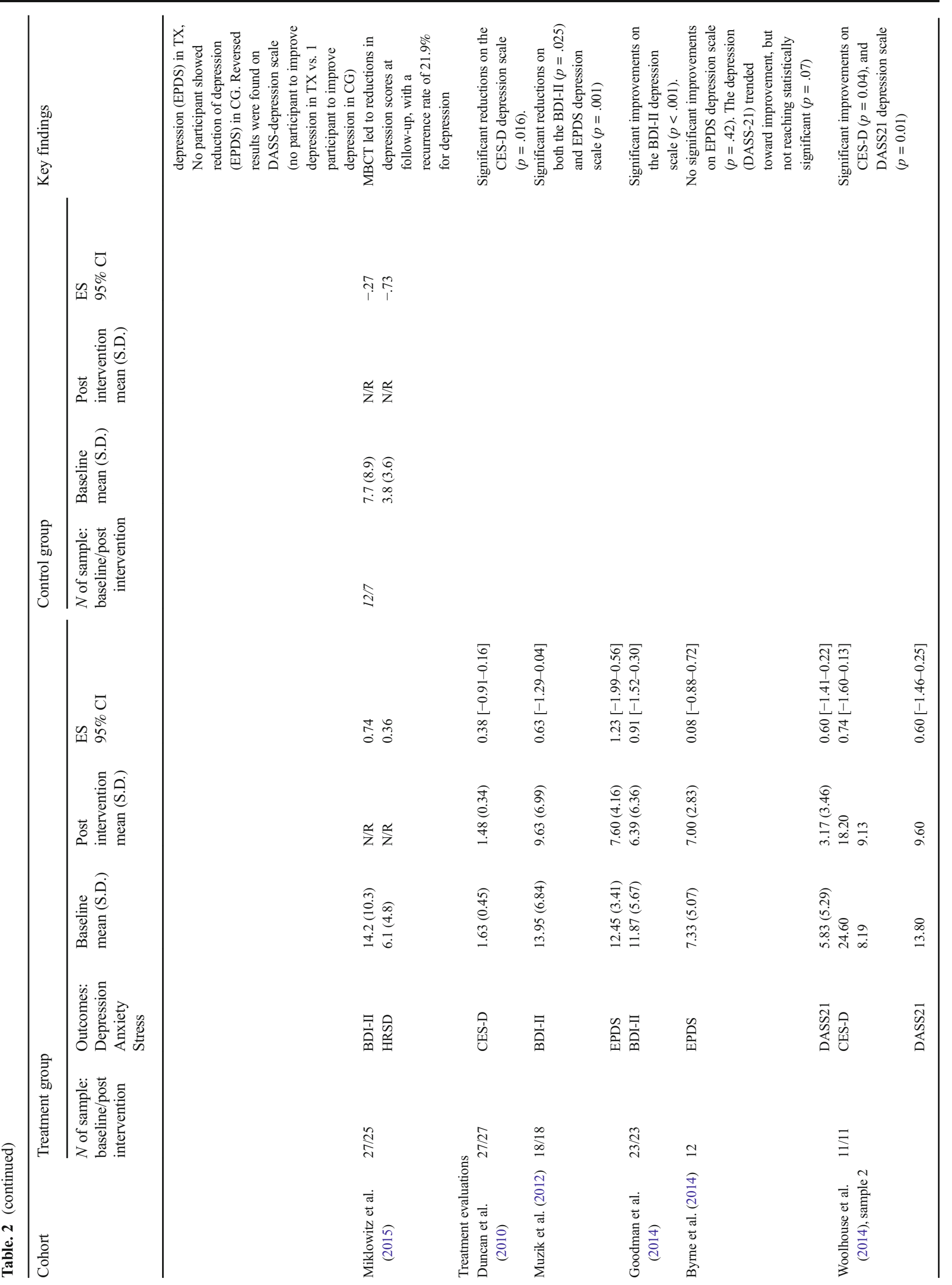




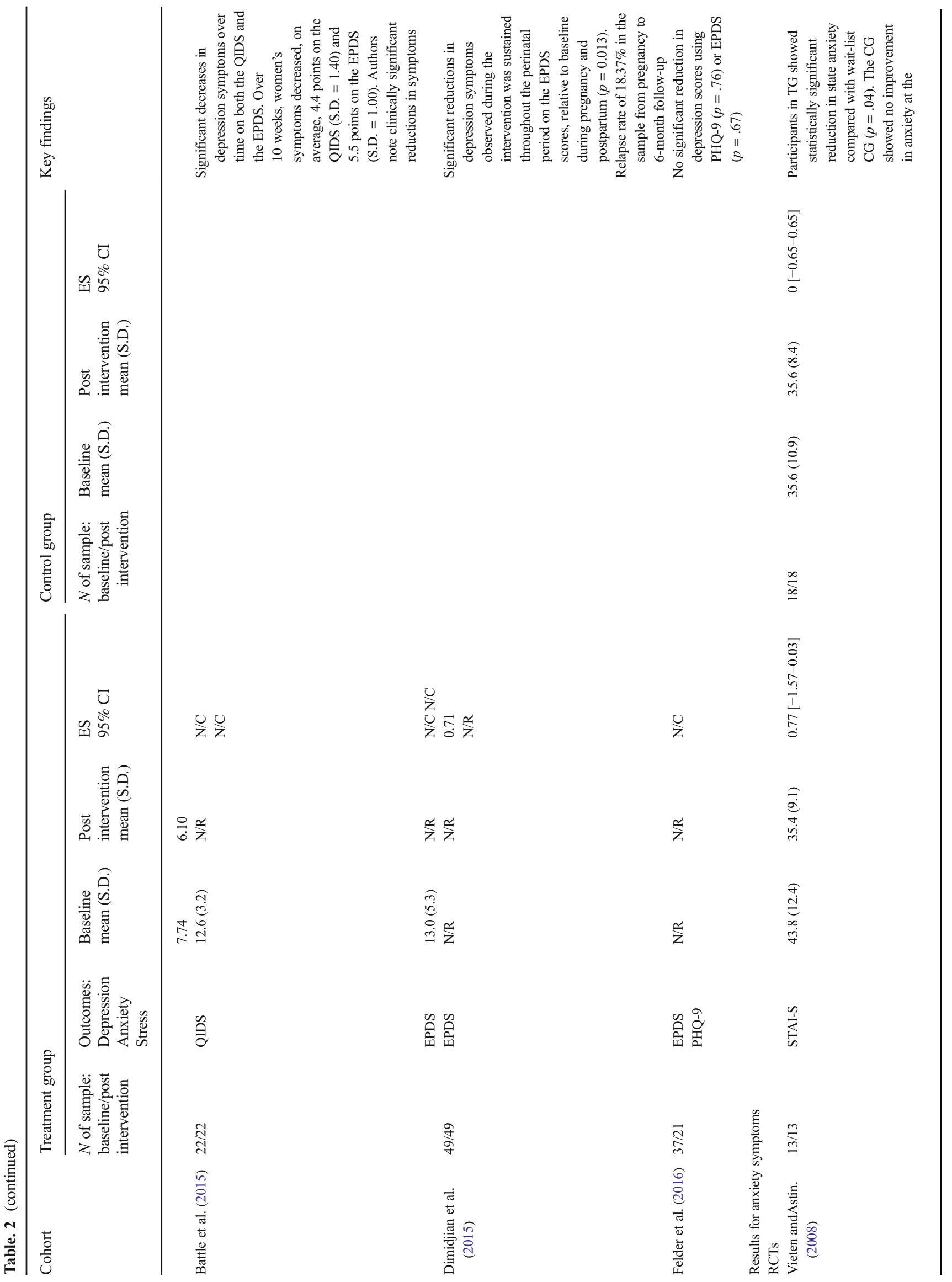




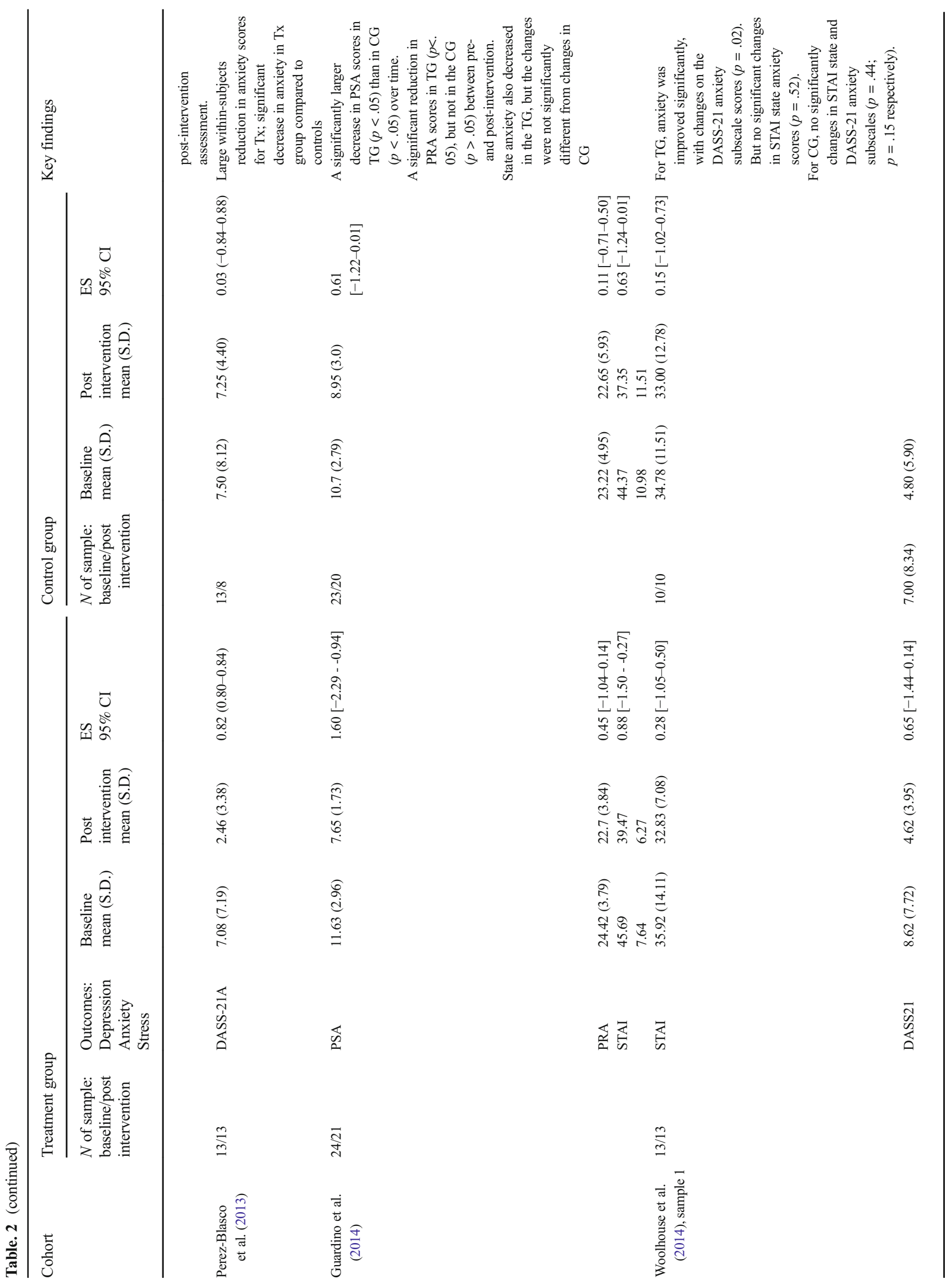




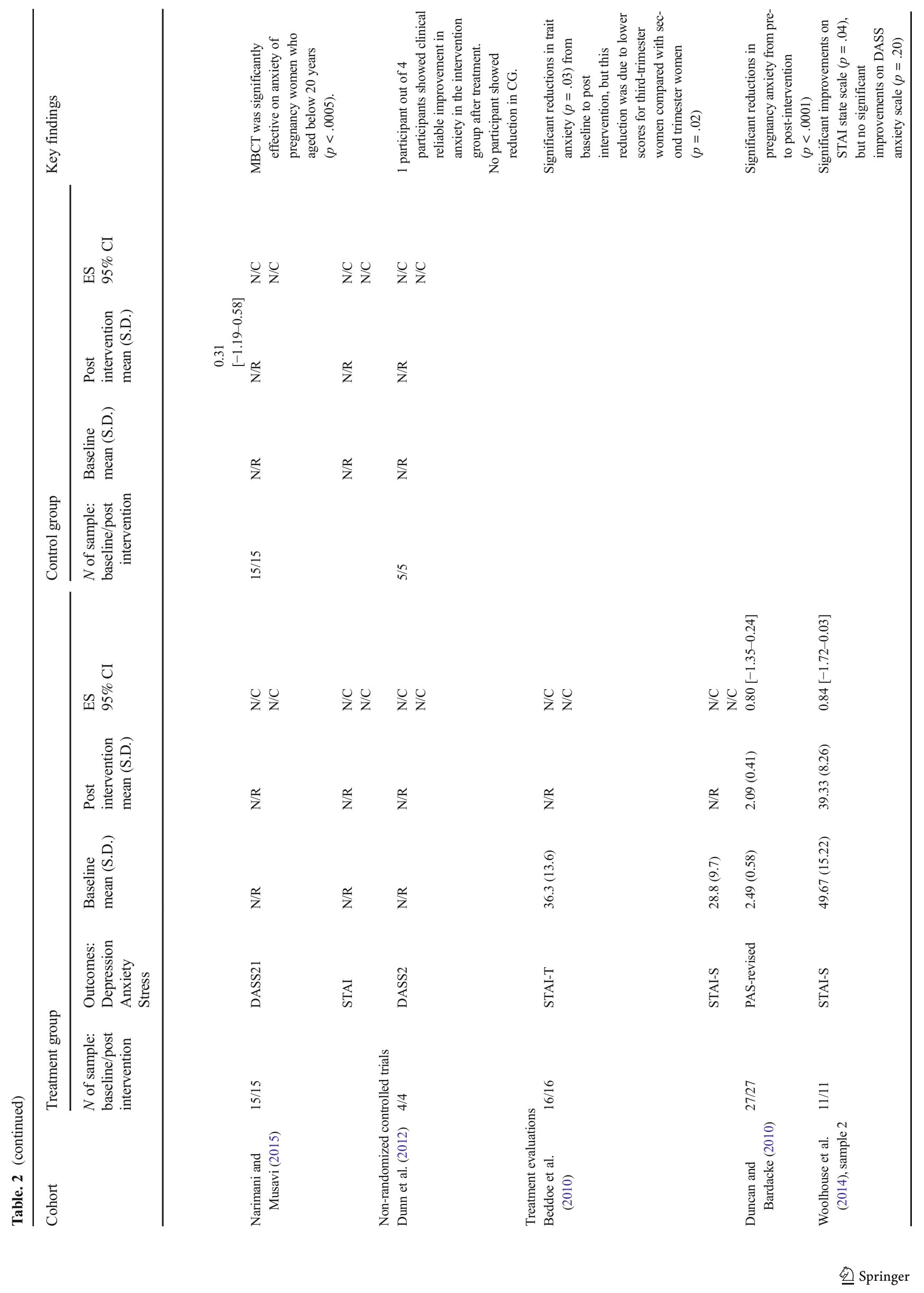




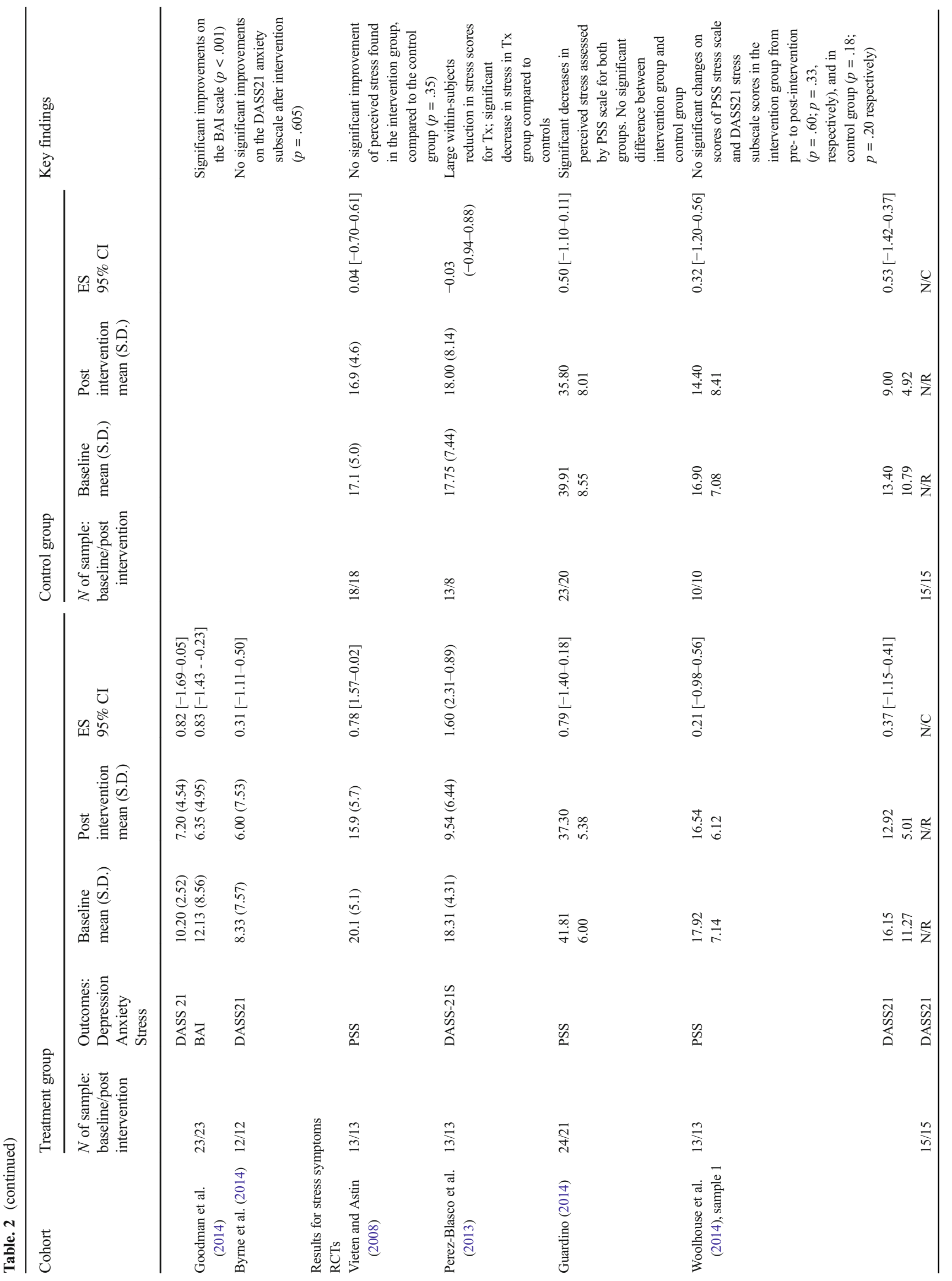




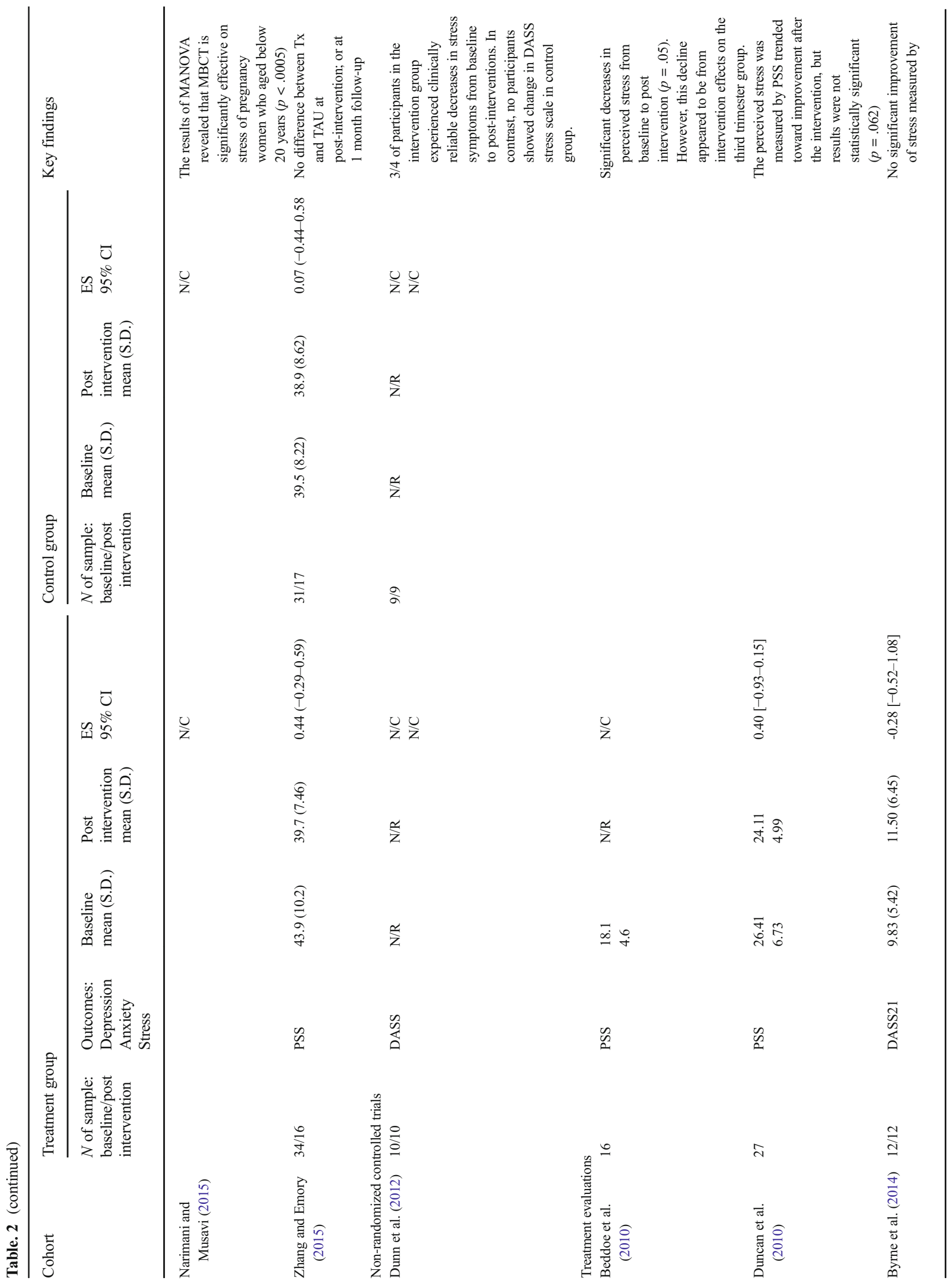




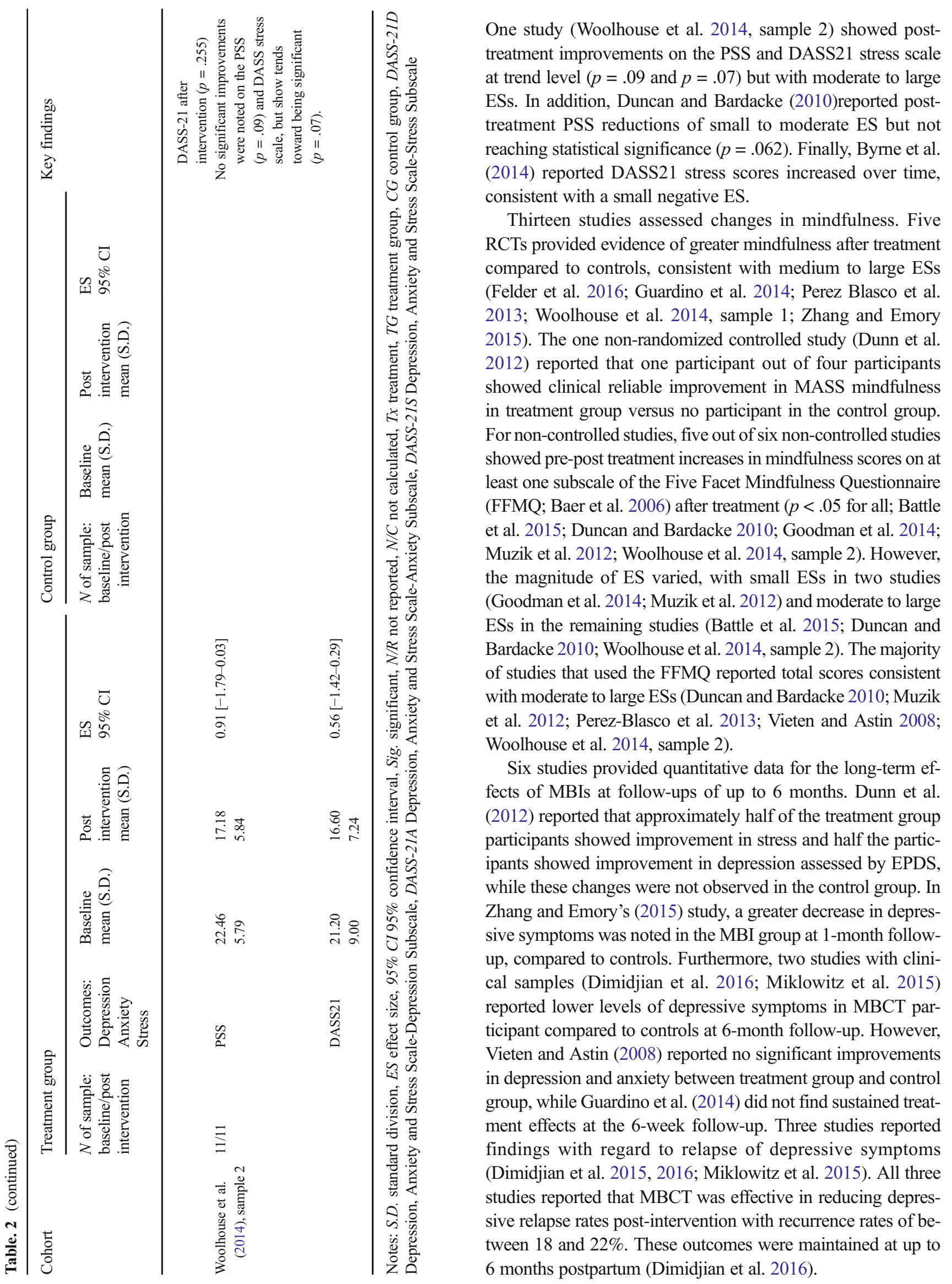




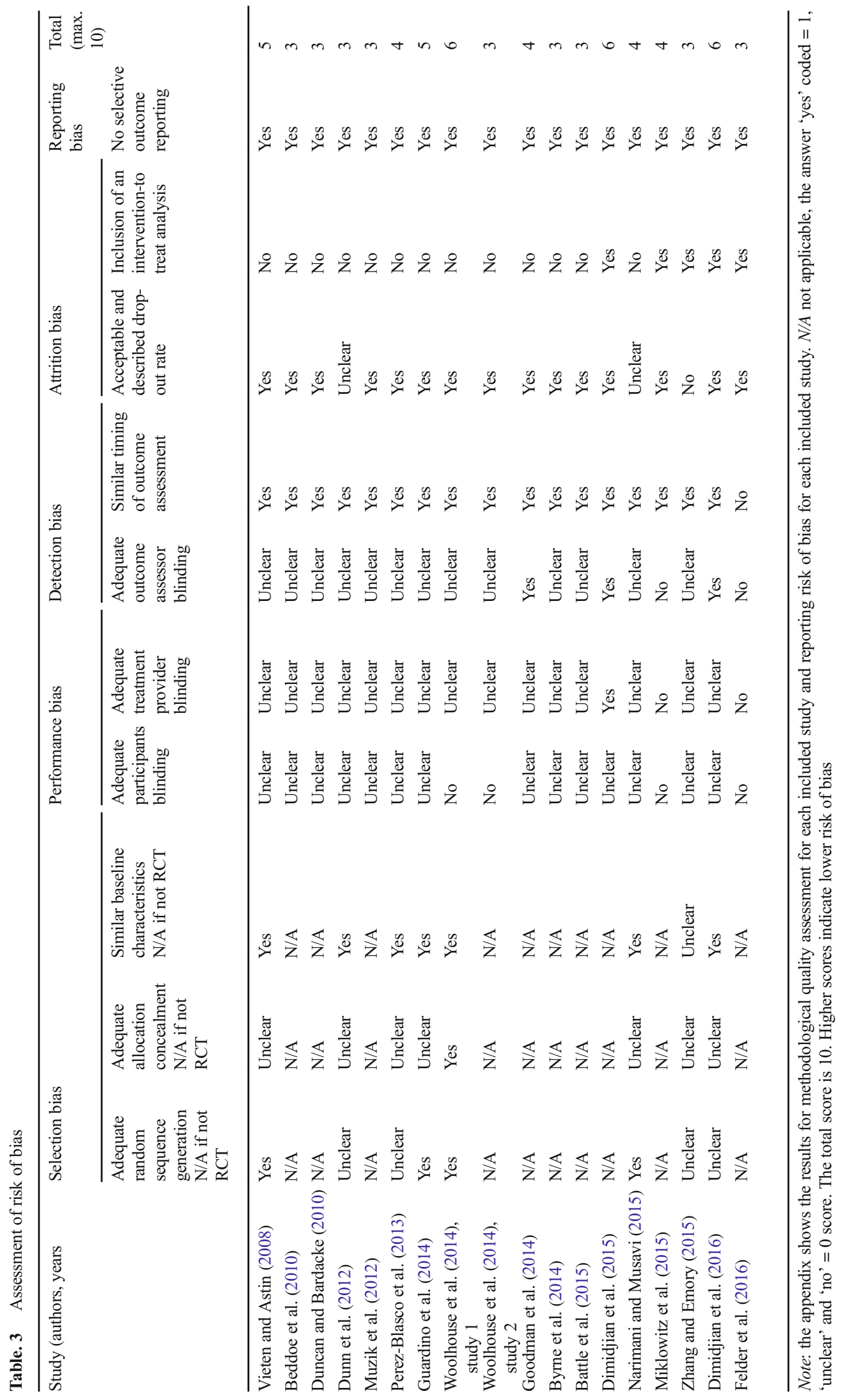


Of the included studies, 16 studies reported the dropout rates. In the RCTs, dropout rates in the treatment group were relatively lower than in the control group, with the exception of one socioeconomic high-risk sample (Zhang and Emory 2015) and an online web program trial (Felder et al. 2016). From the noncontrolled studies, dropout was also relatively low with a range from 4\% (Goodman et al. 2014) to 45\% (Woolhouse et al. 2014, sample 2).

Finally, the results of the risk of bias evaluation are presented in Table 3. Of the 17 included studies, there was considerable variability in the spread of risk of bias ratings, with RCTs reporting greater adherence to attempts to minimize bias. However, there was a spread of ratings with some RCTs having risk of bias ratings similar to non-controlled studies. With regard to methodological aspects, there was evidence that selection bias, performance bias and assessor blinding were generally more consistently omitted or unclear in the included studies. In contrast, all studies reported similar timing of outcome assessments. Most of studies reported dropout rates, and intention-totreat analyses were used in most of the more recent RCTs (Dimidjian et al. 2015, 2016; Miklowitz et al. 2015). Risk of reporting bias was low in all studies.

\section{Discussion}

Our review systematically reviewed the evidence for the effectiveness of MBIs on perinatal depression and anxiety. Outcomes for depression and stress show some evidence of treatment effects, although this was less pronounced in studies comparing MBIs to control groups. The treatment effects of MBIs on anxiety were more consistent and of greater magnitude than the effects of MBIs on depression and stress and were observed across differing study designs. Most studies reported increased mindfulness post treatment, suggesting face validity of the intervention. Although only measured in a minority of studies, there was a small evidence base for the long-term effects of MBIs, particularly in relation to recurrence of depression. This may therefore be a promising avenue for future studies in the area. Taken as a whole, the evidence base suggests that MBIs have high acceptability, as measured by attendance in both general population samples and mental health samples. In the RCT and non-RCT studies, the dropout rates for MBIs appeared lower compared with control groups.

Our findings for anxiety are consistent with previous evidence that MBIs are effective in reducing symptoms of anxiety disorder (e.g. Hofmann et al. 2010; Hoge et al. 2013; Koszycki et al. 2007). It may be the case that mindfulness practice decreases cognitive aspects of anxiety via decreased frequency of negative automatic thoughts (Frewen et al. 2008) or via the impact on physiological arousal. For instance, preliminary evidence suggests that MBIs promote sleep quality for pregnant women (Beddoe et al. 2010). As anxious arousal in the perinatal period may be linked to over-activity of the HPA in infants (Talge et al. 2007), it is also possible that the decreased anxiety associated with MBI may benefit the infant via reduced maternal distress and better regulation of HPA arousal (Salmon et al. 2009).

In contrast to the findings in adult non-pregnant samples (e.g. Hoffman et al. 2010), the review did not find clear associations between MBI and reductions in depressive symptoms. There are several possible explanations for this inconsistent pattern of findings. Under-powering due to small sample size was an issue with several studies showing large but non-significant ESs (e.g. Vieten and Astin 2008). In addition, MBCT was originally designed as an intervention for recurrent depression (Segal 2002). However, most of the samples in the current review had lower levels of baseline depression severity. Therefore, the failure to detect significant change may represent a floor effect. In addition, all reviewed studies that found non-significant treatment effects on depression involved general population samples, with below cutoff scores on depression measures. However, results for noncontrolled studies from perinatal mental health samples showed significant remission of depression after treatment (e.g. Woolhouse et al. 2014). Studies also relied on a diverse range of self-report measures, thus increasing heterogeneity. It is also the case that symptom measures used in the studies may be related to the non-significant outcomes for depression. There also remains the possibility that, despite the sensitivity and specificity of selfreport measures for identifying depression (e.g. Thomas et al. 2001), general measures such as the CES-D may not be adequate to identify depression and anxiety in pregnant or postnatal women, due to the overlap between somatic symptoms of pregnancy and certain items of depression measures (e.g. lack of energy). One alternative would be to use of pregnancy-specific measures of low mood (e.g. EPDS) in conjunction with general measures of depression. We also note that the demographic characteristics of the majority of samples suggested a bias towards relatively welleducated women in stable relationships. This applied to both general population and mental health samples. Therefore, adaptations to the delivery of MBIs may be required to target low-income families or women experiencing multiple adversities.

We acknowledge that the review was limited by the number of studies available and variability in the methodological quality of the primary studies. This heterogeneity led us to focus on a narrative synthesis, rather than conduct a meta-analysis of the results. The included studies varied widely on validation methods, study design, data reporting, severity of mental health difficulties and gestation weeks at baseline, therefore restricting comparisons between studies. We also note that limiting inclusion to studies published in English may have led to the omission of papers. However, Taylor et al. (2016) have recently meta-analyzed the mindfulness studies included in this review. Given the rapid growth of literature in this area, it would be reasonable to conduct a further analysis as the literature increases. We also acknowledge that our review combines samples recruited due to their mental health status and general maternity samples. This 
introduces methodological variance into the synthesis of the results. However, we contend that this ambiguity reflects different care pathways with regard to the assessment and monitoring of mental health in pregnancy, which would be lost with a more stringent focus on inclusion criteria. We also note ambiguity in the primary studies regarding the measurement of depression which was largely based on self-reported depressive symptoms - although the EPDS was used in the majority of studies. Therefore, further research using interview-validated diagnostic measures of low mood would be merited to increase the rigor of assessment of mental health in this area. Furthermore, the review is limited by the lack of follow-up studies to test the long-term effects of MBIs and qualitative results. Finally, we also observe that study risk of bias was variable, but this was not a simple case of all RCTs having reduced bias compared to non-controlled and treatment evaluation studies. Most studies used appropriate analyses, but improvements could be made to the reporting of randomization, blinding and controlling for dropout. We suggest that these difficulties are common across many health service-based treatment evaluations in perinatal and infant mental health (e.g. MacBeth et al. 2015).

In terms of future research and practice, our review highlights the need for more methodologically rigorous trials of MBIs in the perinatal period. This includes greater clarity around the optimal target population for intervention. Much of the research we review used general population samples, suggesting a role for a generalized MBI for wellbeing in pregnancy. However, from a mental health perspective, it may be more effective to target interventions at women meeting 'high-risk' criteria for mental health in pregnancy due to current or previous psychiatric symptoms. Furthermore, trials would benefit from incorporating interviewbased or diagnostic measures of mental disorder into trial protocols. In addition, the role of MBIs in preventing depressive relapse, which was a key driver in the development of MBCT for depression, remains under evaluated. A further consideration with regard to targeting of interventions lies within the differing motivations for engagement with interventions between women from the general population without symptoms of distress compared to women presenting with past or current mental health difficulties. Indeed, the literature on common mental health difficulties in pregnancy highlights that this is likely to be the case (Staneva et al. 2015b). As such, there is scope for qualitative assessment of women's motivations and experiences of MBIs in pregnancy. We also note that the majority of studies were conducted in the USA or Australia. Given the increasing ubiquity of mindfulness practice, it would be beneficial for future studies to be conducted in other settings. Further research is also required with regard to long-term effects of MBIs on maternal and child outcomes. Limitations notwithstanding, our review suggests that MBIs are a non-pharmacological approach to maternal distress likely to be acceptable to women in pregnancy and could therefore be integrated into existing programs of pregnancy care for both with additional targeted adaptations for 'high-risk' groups.

\section{Compliance with Ethical Standards}

Funding No funding to declare. The Paper is based on work submitted as the first author's Masters Dissertation.

Conflicts of Interest Author MacBeth has published on and conducted research on the perinatal psychological needs of women with complex mental health difficulties. He has also published on the effectiveness of third wave psychological interventions. Author Shi has no conflicts of interest.

Open Access This article is distributed under the terms of the Creative Commons Attribution 4.0 International License (http:// creativecommons.org/licenses/by/4.0/), which permits unrestricted use, distribution, and reproduction in any medium, provided you give appropriate credit to the original author(s) and the source, provide a link to the Creative Commons license, and indicate if changes were made.

\section{References}

American Psychiatric Association, (2000). Diagnostic criteria from DSM-IV-TR. American Psychiatric Pub.

Andersson, L., Sundstrom-Poromaa, I., Wulff, M., Astrom, M., \& Bixo, M. (2006). Depression and anxiety during pregnancy and six months postpartum: a follow-up study. Acta Obstetric Gynecologica Scandinavica, 85(8), 937-944.

Baer, R. A., Smith, G. T., Hopkins, J., Krietemeyer, J., \& Toney, L. (2006). Using self-report assessment methods to explore facets of mindfulness. Assessment, 13(1), 27-45.

Battle, C. L., Uebelacker, L. A., Magee, S. R., Sutton, K. A., \& Miller, I. W. (2015). Potential for prenatal yoga to serve as an intervention to treat depression during pregnancy. Women's Health Issues, 25(2), 134-141.

Beck, A. T., \& Steer, R. A. (1990). Manual for the Beck Anxiety Inventory. San Antonio, TX: Psychological Corporation.

Beck, A. T., Steer, R. A., \& Brown, G. K. (1996). Manual for The Beck Depression Inventory Second Edition (BDI-II). San Antonio: Psychological Corporation.

Beddoe, A. E., Lee, K. A., Weiss, S. J., Kennedy, H. P., \& Yang, C. P. P. (2010). Effects of mindful yoga on sleep in pregnant women: a pilot study. Biological Research for Nursing, 11(4), 363-370.

Byrne, J., Hauck, Y., Fisher, C., Bayes, S., \& Schutze, R. (2014). Effectiveness of a mindfulness-based childbirth education pilot study on maternal self-efficacy and fear of childbirth. Journal of Midwifery \& Women's Health, 59(2), 192-197.

Cohen, S., Kamarck, T., \& Mermelstein, R. (1983). A global measure of perceived stress. Journal of Health and Social Behavior, 24(4), 385396.

Cohen, J. (1988). Statistical power analysis for the behavioural sciences. Hillsdale, MI: Lawrence Erlbaum Associates.

Cox, J. L., Holden, J. M., \& Sagovsky, R. (1987). Detection of postnatal depression: development of the 10-item Edinburgh postnatal depression scale. British Journal of Psychiatry, 150(6), 782-786.

Cramer, H., Lauche, R., \& Dobos, G. (2013). Yoga for depression: a systematic review and meta-analysis. Depression \& Anxiety, 30(11), 1068-1083.

Curtis, K., Weinrib, A., \& Katz, J. (2012). Systematic review of yoga for pregnant women: current status and future directions. Evidencebased complementary and alternative medicine

Di Florio, A., Forty, L., Gordon-Smith, K., Heron, J., Jones, L., Craddock, N., et al. (2013). Perinatal episodes across the mood disorder spectrum. JAMA Psychiatry, 70(2), 168-175. 
Dimidjian, S., Goodman, S. H., Felder, J. N., Gallop, R., Brown, A. P., \& Beck, A. (2015). An open trial of mindfulness-based cognitive therapy for the prevention of perinatal depressive relapse/recurrence. Archives of Women's Mental Health, 18(1), 85-94.

Dimidjian, S., Goodman, S. H., Felder, J. N., Gallop, R., Brown, A. P., \& Beck, A. (2016). Staying well during pregnancy and the postpartum: a pilot randomized trial of mindfulness-based cognitive therapy for the prevention of depressive relapse/recurrence. Journal of Consulting and Clinical Psychology, 84(2), 134.

Dole, N., Savitz, D. A., Hertz-Picciotto, I., Siega-Riz, A. M., McMahon, M. J., \& Buekens, P. (2003). Maternal stress and preterm birth. American Journal of Epidemiology, 157(1), 14-24.

Duncan, L. G., \& Bardacke, N. (2010). Mindfulness-based childbirth and parenting education: promoting family mindfulness during the perinatal period. Journal of Child and Family Studies, 19(2), 190-202.

Dunkel Schetter, C., \& Lobel, M. (2012). Pregnancy and birth: a multilevel analysis of stress and birth weight. In T. A. Revenson, A. Baum, \& J. Singer (Eds.), Handbook of health psychology (2nd ed., pp. 427-453). London: Psychology Press.

Dunn, C., Hanieh, E., Roberts, R., \& Powrie, R. (2012). Mindful pregnancy and childbirth: effects of a mindfulness based intervention on women's psychological distress and well-being in the perinatal period. Archives of Women's Menalt Health, 15(2), 139-143.

Felder, J.N., Segal, Z., Beck, A., Sherwood, N.E., Goodman, S.H., Boggs, J., Lemon, E. and Dimidjian, S., (2016). An open trial of web-based mindfulness-based cognitive therapy for perinatal women at risk for depressive relapse. Cognitive and behavioral practice.

Field, T., Diego, M., Hernandez-Reif, M., Figueiredo, B., Deeds, O., Ascencio, A., et al. (2010). Comorbid depression and anxiety effects on pregnancy and neonatal outcome. Infant Behavioral Development, 33(1), 23-29.

Frewen, P., Evans, E., Maraj, N., Dozois, D., \& Partridge, K. (2008). Letting go: mindfulness and negative automatic thinking. Cognitive Therapy Research, 32(6), 758-774.

Glover, V., \& O'Connor, T. (2006). Maternal anxiety: its effect on the fetus and the child. British Journal of Midwifery, 14(11), 663-667.

Glynn, L. M., Schetter, C. D., Hobel, C. J., \& Sandman, C. A. (2008). Pattern of perceived stress and anxiety in pregnancy predicts preterm birth. Health Psychology, 27(1), 43-51.

Gong, H., Ni, C., Shen, X., Wu, T., \& Jiang, C. (2015). Yoga for prenatal depression: a systematic review and meta-analysis. BMC Psychiatry, 15(1), 14-22.

Goodman, J. H., \& Tyer-Viola, L. (2010). Detection, treatment, and referral of perinatal depression and anxiety by obstetrical providers. Journal of Women's Health, 19(3), 477-490.

Goodman, J. H., Guarino, A., Chenausky, K., Klein, L., Prager, J., Petersen, R., et al. (2014). CALM pregnancy: results of a pilot study of mindfulness-based cognitive therapy for perinatal anxiety. Archives of Women's Mental Health, 17(5), 373-387.

Grigoriadis, S., de Camps, M. D., Barrons, E., Bradley, L., Eady, A., Fishell, A., et al. (2011). Mood and anxiety disorders in a sample of Canadian perinatal women referred for psychiatric care. Archives of Women's Mental Health, 14(4), 325-333.

Grigoriadis, S., VonderPorten, E. H., Mamisashvili, L., Tomlinson, G., Dennis, C.-L., Koren, G., Steiner, M., Mousmanis, P., Cheung, A., \& Ross, L. E. (2014). Prenatal exposure to antidepressants and persistent pulmonary hypertension of the newborn: systematic review and meta-analysis. BMJ, 348. doi:10.1136/bmj.f6932.

Guardino, C. M., Schetter, C. D., Bower, J. E., Lu, M. C., \& Smalley, S. L. (2014). Randomised controlled pilot trial of mindfulness training for stress reduction during pregnancy. Psychology \& Health, 29(3), 334-349.

Hall, H. G., Beattie, J., Lau, R., East, C., \& Biro, M. A. (2016). Mindfulness and perinatal mental health: a systematic review. Women and Birth, 29(1), 62-71.
Hann, D., Winter, K., \& Jacobsen, P. (1999). Measurement of depressive symptoms in cancer patients. Evaluation of the Center for Epidemiological Studies Depression Scale (CES-D). Journal of Psychosomatic Research, 46(5), 437-443.

Hendrick, V., Smith, L. M., Suri, R., Hwang, S., Haynes, D., \& Altshuler, L. (2003). Birth outcomes after prenatal exposure to antidepressant medication. American. Journal. Of Obstetrics \&. Gynecology, 188(3), 812-815.

Higgins, J. P., Altman, D. G., Gøtzsche, P. C., Jüni, P., Moher, D., Oxman, A. D., Savović, J., Schulz, K. F., Weeks, L., \& Sterne, J. A. (2011). The Cochrane Collaboration's tool for assessing risk of bias in randomised trials. $B M J, 343,5928$.

Hoffman, S. G., \& Hatch, M. C. (2000). Depressive symptomatology during pregnancy: evidence for an association with decreased fetal growth in pregnancies of lower social class women. Health Psychology, 19(6), 535-543.

Hoffman, S. G., Sawyer, A. T., Witt, A. A., \& Oh, D. (2010). The effect of mindfulness-based therapy on anxiety and depression: a metaanalytic review. Journal of Consulting and Clinical Psychology, 78(2), 169-183.

Hoge, E. A., Bui, E., Marques, L., Metcalf, C. A., Morris, L. K., Robinaugh, D. J., et al. (2013). Randomized controlled trial of mindfulness meditation for generalized anxiety disorder: effects on anxiety and stress reactivity. Journal of Clinical Psychiatry, 74(8), 786792.

Huang, H., Coleman, S., Bridge, J. A., Yonkers, K., \& Katon, W. (2014). A meta-analysis of the relationship between antidepressant use in pregnancy and the risk of preterm birth and low birth weight. General Hospital Psychiatry, 36(1), 13-18.

Huizink, A. C., Robles de Medina, P. G., Mulder, E. J., Visser, G. H., \& Buitelaar, J. K. (2003). Stress during pregnancy is associated with developmental outcome in infancy. Journal of Child Psychology and Psychiatry, 44(6), 810-818.

Kabat-Zinn, J. (2003). Mindfulness-based interventions in context: past, present, and future. Clinical Psychology: Science and Practice, $10(2), 144-156$

Kallen, B. (2004). Neonate characteristics after maternal use of antidepressants in late pregnancy. Archives of Pediatric and Adolescent Medicine, 158(4), 312-316.

Koszycki, D., Benger, S. J., \& Bradwejn, J. (2007). Randomized trial of a meditation-based stress reduction program and cognitive behavior therapy in generalized social anxiety disorder. Behavior Research and Therapy, 45(10), 2518-2526.

Kuyken, W., Hayes, R., Barrett, B., Byng, R., Dalgleish, T., Kessler, D., Lewis, G., Watkins, E., Brejcha, C., Cardy, J., \& Causley, A. (2015). Effectiveness and cost-effectiveness of mindfulness-based cognitive therapy compared with maintenance antidepressant treatment in the prevention of depressive relapse or recurrence (PREVENT): a randomised controlled trial. The Lancet, 386(9988), 63-73.

Levin, J. S. (1991). The factor structure of the pregnancy anxiety scale. Journal of Health and Social Behavior, 32(4), 368-381.

Leung, B. M., \& Kaplan, B. J. (2009). Perinatal depression: prevalence, risks, and the nutrition link-a review of the literature. Journal of the American Dietetic Association, 109(9), 1566-1575.

Lovibond, P. F., \& Lovibond, S. H. (1995). The structure of negative emotional states: comparison of the Depression Anxiety Stress Scales (DASS) with the Beck Depression and Anxiety Inventories. Behavior Research and Therapy, 33(3), 335-342.

MacBeth, A., Law, J., McGowan, I., Norrie, J., Thompson, L., \& Wilson, P. (2015). Mellow parenting: systematic review and meta-analysis of an intervention to promote sensitive parenting. Developmental Medicine \& Child Neurology, 57(12), 1119-1128.

Marteau, T., \& Bekker, H. (1992). The development of a six-item shortform of the state scale of the Spielberger State-Trait Anxiety Inventory (STAI). British Journal of Psychology, 31(3), 301-306. 
Miklowitz, D. J., Semple, R. J., Hauser, M., Elkun, D., Weintraub, M. J., \& Dimidjian, S. (2015). Mindfulness-based cognitive therapy for perinatal women with depression or bipolar spectrum disorder. Cognitive Therapy and Research, 39(5), 590-600.

Moher, D., Shamseer, L., Clarke, M., Ghersi, D., Liberati, A., Petticrew, M., Shekelle, P., \& Stewart, L. A. (2015). Preferred reporting items for systematic review and meta-analysis protocols (PRISMA-P) 2015 statement. Systematic reviews, 4(1), 1.

Muzik, M., Hamilton, S. E., Rosenblum, K. L., Waxler, E., \& Hadi, Z. (2012). Mindfulness yoga during pregnancy for psychiatrically atrisk women: preliminary results from a pilot feasibility study. Complementary Therapies in Clinical Practice, 18(4), 235-240.

Narendran, S., Nagarathna, R., Narendran, V., Gunasheela, S., \& Nagendra, H. R. R. (2005). Efficacy of yoga on pregnancy outcome. Journal of Alternative \& Complementary Medicine, 11(2), 237-244.

Narimani, M., \& Musavi, S. K. S. (2015). Effectiveness of mindfulness based cognitive therapy on anxiety, stress and depression of pregnant adolescents: a randomized clinical trial. European Journal of Forensic Sciences, 2(1), 1-4.

Newham, J. J., Wittkowski, A., Hurley, J., Aplin, J. D., \& Westwood, M. (2014). Effects of antenatal yoga on maternal anxiety and depression: a randomized controlled trial. Depression and Anxiety, 31(8), 631-640.

Nicol-Harper, R., Harvey, A. G., \& Stein, A. (2007). Interactions between mothers and infants: impact of maternal anxiety. Infant Behavioral Development, 30(1), 161-167.

O'Connor, T. G., Heron, J., \& Glover, V. (2002). Antenatal anxiety predicts child behavioural/emotional problems independently of postnatal depression. Journal of the American Academy of Child and Adolescent Psychiatry, 41(12), 1470-1477.

Perez-Blasco, J., Viguer, P., \& Rodrigo, M. F. (2013). Effects of a mindfulness-based intervention on psychological distress, well-being, and maternal self-efficacy in breast-feeding mothers: results of a pilot study. Archives of women's mental health, 16, 227-236.

Piet, J., \& Hougaard, E. (2011). The effect of mindfulness-based cognitive therapy for prevention of relapse in recurrent major depressive disorder: a systematic review and meta-analysis. Clinical Psychology Review, 31(6), 1032-1040.

Quevedo, L. A., Silva, R. A., Godoy, R., Jansen, K., Matos, M. B., Tavares Pinheiro, K. A., et al. (2012). The impact of maternal post-partum depression on the language development of children at 12 months. Child Care and Health Development, 38(3), 420-424.

Radloff, L. S. (1977). The CED-D scale: a self-report depression scale for research in the general population. Applied Psychological Measurement, 1, 385-401.

Rush, A. J., Bernstein, I. H., Trivedi, M. H., Carmody, T. J., Wisniewski, S., Mundt, J. C., et al. (2006). An evaluation of the quick inventory of depressive symptomatology and the Hamilton rating scale for depression: a sequenced treatment alternatives to relieve depression trial report. Biological Psychiatry, 59(6), 493-501.

Salmon, P., Lush, E., Jablonski, M., \& Sephton, S. E. (2009). Yoga and mindfulness: clinical aspects of an ancient mind/body practice. Cognitive and Behavioral Practice, 16(1), 59-72.
Scottish Intercollegiate Guideline Network checklist. Edinburgh (2015): http://www.sign.ac.uk/methodology/checklists.html

Segal, Z. V., Williams, J. M. G., \& Teasdale, J. D. (2002). Preventing depression: mindfulness-based cognitive therapy. New York: Guilford.

Spielberger, C. D. (1989). State-Trait Anxiety Inventory: Bibliography (2nd ed.). Palo Alto, CA: Consulting Psychologists Press

Staneva, A., Bogossian, F., Pritchard, M., \& Wittkowski, A. (2015b). The effects of maternal depression, anxiety, and perceived stress during pregnancy on preterm birth: a systematic review. Women and Birth, 28(3), 179-193.

Staneva, A. A., Bogossian, F., \& Wittkowski, A. (2015a). The experience of psychological distress, depression, and anxiety during pregnancy: a meta-synthesis of qualitative research. Midwifery, 31(6), 563-573.

Sutter-Dallay, A. L., Giaconne-Marcesche, V., Glatigny-Dallay, E., \& Verdoux, H. (2004). Women with anxiety disorders during pregnancy are at increased risk of intense postnatal depressive symptoms: a prospective survey of the MATQUID cohort. European Psychiatry, 19(8), 459-463.

Sydsjö, G. (2011). Long-term consequences of non-optimal birth characteristics. American Journal of Reproductive Immunology, 66(s1), $81-87$.

Talge, N. M., Neal, C., Glover, V., \& the Early Stress, Translational Research and Prevention Science Network: Fetal and Neonatal Experience on Child and Adolescent Mental Health. (2007). Antenatal maternal stress and long-term effects on child neurodevelopment: how and why? Journal of Child Psychology and Psychiatry, 48(3-4), 245-261.

Taylor, B. L., Cavanagh, K., \& Strauss, C. (2016). The effectiveness of mindfulness-based interventions in the perinatal period: a systematic review and meta-analysis. PloS One, 11(5), e0155720.

Thomas, J. L., Jones, G. N., Scarinci, I. C., Mehan, D. J., \& Brantley, P. J. (2001). The utility of the CES-D as a depression screening measure among low-income women attending primary care clinics. The International Journal of Psychiatry in Medicine, 31(1), 25-40.

Wilson-Costello, D., Friedman, H., Minich, N., Fanaroff, A. A., \& Hack, M. (2005). Improved survival rates with increased neurodevelopmental disability for extremely low birth weight infants in the 1990s. Pediatrics, 115(4), 997-1003.

Woolhouse, H., Mercuri, K., Judd, F., \& Brown, S. J. (2014). Antenatal mindfulness intervention to reduce depression, anxiety and stress: a pilot randomized controlled trial of the MindBabyBody program in an Australian tertiary maternity hospital. BMC Pregnancy and Childbirth, 14, 369-397.

World Health Organization, (1993). The ICD-10 classification of mental and behavioural disorders: diagnostic criteria for research.

Vieten, C., \& Astin, J. (2008). Effects of a mindfulness-based intervention during pregnancy on prenatal stress and mood: results of a pilot study. Archives of Women's Mental Health, 11(1), 67-74.

Zhang, H., \& Emory, E. K. (2015). A mindfulness-based intervention for pregnant African-American women. Mindfulness, 6(3), 663-674. 